\title{
An Algebraic Approach to Unital Quantities and their Measurement
}

\author{
Invited paper
}

\author{
Zoltan Domotor ${ }^{1}$ and Vadim Batitsky ${ }^{2}$ \\ ${ }^{1}$ Departments of Philosophy, Biochemistry and Biophysics, University of Pennsylvania, 433 Cohen Hall, 249 South $36^{\text {th }}$ Street, \\ Philadelphia, PA 19104-6304,USA, zdomotor@sas.upenn.edu \\ ${ }^{2}$ Department of Philosophy, St John's University, 800 Utopia Parkway, Queens, NY 11439, USA
}

\begin{abstract}
The goals of this paper fall into two closely related areas. First, we develop a formal framework for deterministic unital quantities in which measurement unitization is understood to be a built-in feature of quantities rather than a mere annotation of their numerical values with convenient units. We introduce this idea within the setting of certain ordered semigroups of physical-geometric states of classical physical systems. States are assumed to serve as truth makers of metrological statements about quantity values. A unital quantity is presented as an isomorphism from the target system's ordered semigroup of states to that of positive reals. This framework allows us to include various derived and variable quantities, encountered in engineering and the natural sciences. For illustration and ease of presentation, we use the classical notions of length, time, electric current and mean velocity as primordial examples. The most important application of the resulting unital quantity calculus is in dimensional analysis. Second, in evaluating measurement uncertainty due to the analog-to-digital conversion of the measured quantity's value into its measuring instrument's pointer quantity value, we employ an ordered semigroup framework of pointer states. Pointer states encode the measuring instrument's indiscernibility relation, manifested by not being able to distinguish the measured system's topologically proximal states. Once again, we focus mainly on the measurement of length and electric current quantities as our motivating examples. Our approach to quantities and their measurement is strictly state-based and algebraic in flavor, rather than that of a representationalist-style structure-preserving numerical assignment.
\end{abstract}

Keywords: Deterministic measurement, measurement unit, measurement uncertainty, pointer quantity, pointer state, quantity calculus, quantization, state space, unital quantity.

\section{INTRODUCTION AND OVERVIEW}

A natural starting point for the investigation of quantities and their measurement in the natural sciences and engineering is the development of a conceptual framework that effectively facilitates the basic numerical ways of expressing scientific knowledge and inferential reasoning about the structure and behavior of systems of interest. Quantities and their measurements are known to be studied within the settings of several competing theories from different perspectives and at different levels of detail.

\subsection{The status of deterministic quantity calculus in natural sciences}

Historians of metrology (see, e.g., [2]) tend to attribute the first systematic account of the notion of deterministic physical quantity to James Clerk Maxwell. In his often cited 1873 monograph Treatise on Electricity and Magnetism (Oxford University Press), Maxwell in essence states the following about characterizing a quantity: Every expression of a quantity consists of two factors. One of these is the name of a certain known quantity (a standard of reference) of the same DOI: $10.1515 / \mathrm{msr}-2016-0014$ kind as the quantity to be expressed, and the other is the number of times the standard is to be taken in order to make up the target quantity, known as the quantity value. With the symbolization commonly used and recommended by the International Standards Organization [9], Maxwell's description of a physical quantity $Q$ is expressed by the equational formula

$$
Q=\{Q\} \cdot[Q]
$$

in which $\{Q\}$ denotes the numerical value of quantity $Q$ and $[Q]$ signifies the reference quantity or measurement unit associated with $Q$. Maxwell's formula is easier to comprehend in terms of concrete examples. For instance, we have the familiar metrological assertion "length $(\mathfrak{D})=7.5 \mathrm{~m}$ " about the length of an unspecified length-bearing object $\mathfrak{o}$ (e.g., a flagpole), expressed in the traditional meter measurement unit. Likewise, the statement "mass $(\mathfrak{p})=10.3 \mathrm{~kg}$ " is about the rest mass of an unspecified mass-bearing object o (e.g., a cinder block localized in a Newtonian reference frame) formulated in the kilogram unit of measure. Because in these statements there is no question-begging multiplication symbol, some metrologists prefer to formulate Maxwell's equa- 
tion without it and simply write $Q=\{Q\}[Q] .{ }^{1}$ To avoid certain dimension-theoretic ambiguities, suggestions have also been made to use a three-factor expression of the form

$$
Q=\{Q\} \cdot[Q] \cdot \operatorname{dim} Q
$$

where $\operatorname{dim} Q$ denotes the physical dimension of quantity $Q$. The advocates of this somewhat refined approach to analyzing the concept of quantity do not specify the properties of product operations used in the tripartite facorization. Hurdles generated by the kind of quantities (an aspect common to mutually compatible quantities) are overcome by adding a fourth factor kind $Q$ to the foregoing formula, intended for specifying the kind of quantity $Q$. Obviously, the growing number factors begins to look rather tedious. Algebraic studies of these constructions might help metrologists to understand some of the pittfals that may attend their efforts in isolating the correct notion of a single-valued deterministic quantity.

Since in Maxwell's equation the factor [Q] is widely thought to be just another quantity and $\{Q\}$ refers to a real number, it is puzzling why the equation above is not stated as a simple quantity conversion principle: For any given quantity $Q$ and a designated reference quantity $Q^{\prime}$ of the same kind there exists exactly one positive real number $\alpha$ such that $Q=\alpha \cdot Q^{\prime}{ }^{2}$ Therefore $\alpha$ can be thought of as being uniquely specified by the numerical ratio $\frac{Q}{Q^{\prime}}$ of $Q$ to $Q^{\prime}$. ${ }^{3}$

And of course to any given quantity $Q$ and a strictly positive real number $\alpha$ there exists a unique quantity $Q^{\prime}$ of the same kind such that the scalar product equation $Q=\alpha \cdot Q^{\prime}$ holds. Since Maxwell's quantity $Q$ does not possess an intrinsic numerical value or an inherent reference quantity, it is important to keep in mind that the curly-bracket notation $\{Q\}$ for $Q$ 's numerical values is empirically meaningless in the absence of the associated unit quantity $[Q]$. To be meaningful, these two expressions must always be used together. It should be remarked that treating the square bracket symbolism as a legitimate mapping of quantities to quantities is a serious misconception.

The most obvious way to handle this sort of metrological codependent relationship is to work with "unital quantities" in which the unit is a built-in intrinsic feature of quantities, somewhat akin to formulations used in quantitative assertions about, say, the length of a beam: "length ${ }_{\mathrm{m}}($ beam $)=6.5$ ", where the indexing unit symbol $\mathrm{m}$ refers to the incorporated meter unit of measure. Simple examples of unital length quantities are length-in-meters, length-in-feet, etc.. Common examples of unital mass quantities are mass-in-kilograms, mass-in-pounds, and so forth. Experimenters are likely to

\footnotetext{
${ }^{1}$ Even if the symbol for the scalar product is omitted, it is implied.

${ }^{2}$ Although in the case of extensive quantities, such as length, time, mass, and electric current, the standard requirement is that their numerical values be positive real numbers forming the set $\mathbb{R}_{+}$, there are obvious generalizations to quantities that permit arbitrary real or complex number values. However, it does not seem important to examine these technical variations here, except perhaps the set $\mathbb{R}_{+0}$ of positive real nunbers including zero.

${ }^{3}$ The numerical ratio must not be confused with the derived product quantity $Q \cdot \frac{1}{Q^{\prime}}$ (e.g., mean velocity, defined by distance over time) we discuss below.
}

use measurement units that are strictly tailored to the phenomena they study. Thus, on this approach, when we measure, for example, the length of a rigid metal beam, it is not that we contemplate a particular measurement unit with which to measure the beam's unique quantitative attribute called 'length', we simply choose to measure a particular unital length of the beam, antecendently determined by a calibrated meter stick or some other appropriate length measuring instrument, used in performing the measurement operation. When recording measurement results for statistical analysis, it is clearly simpler to record the numerical interval (say) $6.5 \pm 0.05$ for the beam's possible length-in-meters than working with denominate numbers as in "The beam's length $=6.5$ meters \pm 0.05 meters."

What we take to be pivotal for the unital quantity concept is its unique trait that automatically specifies the accompanying measurement unit, without the usual indexing or the "-in-" suffixal qualifier. It should be remarked that this kind of unital-quantity expression is regularly used in labeling the axes of graphs and headings of table columns. Those with qualms about treating units of measure in terms of unitized quantities can rest assured that this approach is (as we shall show in subsequent sections) significantly superior to Maxwell's characterization.

Our central point about Maxwell's formula is this: since according to metrologists the most striking feature of classical measurement is the estimation of ratios of quantities, the formula does not provide a sufficiently convenient instrument for everyday formulations of empirical claims about quantity values. In parallel to Maxwell's product expression we should also use the ratio counterpart expression, introduced by the one-to-one correspondence

$$
\frac{Q}{Q_{\mathrm{u}}}=\{Q\} \Longleftrightarrow Q=\{Q\} \cdot Q_{\mathrm{u}}
$$

where $Q_{u}$ denotes the reference quantity, specified by a chosen unit $u$ we shall discuss later. A major benefit of the ratio approach is that it provides direct access to the rich structure of real numbers. In particular, we have the obvious multiplication law $\frac{Q}{Q_{u}} \cdot \frac{Q_{u}}{Q_{v}}=\frac{Q}{Q_{v}}$, capturing the change of quantity values obtained by passing from unit $u$ to unit v. And of course we have the trivial identity condition: if $\frac{Q}{Q_{u}}=1$, then $Q=Q_{u}$. The notion of ratios is designed to accommodate Maxwell's idea as its close cousin. Specifically, we now have the equality

$$
Q=\frac{Q}{Q^{\prime}} \cdot Q^{\prime}
$$

that circumvents the above-discussed ambiguity in Maxwell's formula.

The most popular argument against the ratio-based formulation of Maxwell's equation centers around the fact that ratios of pairs of quantities of the same kind are real numbers. Therefore, e.g., a ratio of two length quantities may turn out to be equal to the ratio of two mass quantities. This seemingly troubling illegitimacy of equating length values with mass values is quickly removed by imposing a type-theoretic 
condition, stating that the spaces of length and mass quantities are disjoint. Concretely, from a formal standpoint we may come across the numerical equality

$$
\frac{Q}{Q_{\mathrm{u}}}=\frac{Q^{\prime}}{Q_{\mathrm{u}^{\prime}}^{\prime}}
$$

for some pair of distinct quantities $Q$ and $Q^{\prime}$, but notice that the ratio operations on the left and right sides of the equation are different, symbolized by the increased thickness of the fraction line on the right. So then, because the ratio calculus is context-dependent on the space of quantities under consideration, the other ratios are automatically screened off as empirically meaningless. In general, the difficulties that beset ratio calculus arise from failing to recognize that each quantity of a given kind is accompanied by its own quantity space and structure thereon, so that even if some numerical values of two quantities of a different kind happen to be the same, their algebraic structures and associated operations, and therefore semantics, remain different.

In this paper we develop a robust and algebraically attractive framework for quantities and quantity calculus that directly addresses the issue of ratios. For example, based on ratios of quantities of the same kind, their addition may be formulated quite simply as follows:

$$
Q+Q^{\prime}=d f\left(\frac{Q}{Q^{\prime \prime}}+\frac{Q^{\prime}}{Q^{\prime \prime}}\right) \cdot Q^{\prime \prime}=\left(1+\frac{Q}{Q^{\prime}}\right) \cdot Q^{\prime}
$$

Here and below we use the symbol $=_{d f}$ to indicate equality by definition. ${ }^{4}$

Along similar lines, the natural "less than" total order relation on positive real numbers automatically transfers to quantities by setting $Q<Q^{\prime}$ just in case $\frac{Q}{Q^{\prime \prime}}<\frac{Q^{\prime}}{Q^{\prime \prime}}$ for any reference quantity $Q^{\prime \prime}$. The total order relation is called "natural" because it satisfies the solvability condition

$$
Q<Q^{\prime} \Longleftrightarrow Q+Q^{\prime \prime}=Q^{\prime}
$$

for some quantity $Q^{\prime \prime}$. We shall also provide a closely related definition of partial subtraction $Q-Q^{\prime}$, which is well-defined only for quantity pairs satisfying $Q^{\prime}<Q$.

There is widespread agreement amongst metrologists that a careful distinction needs to be made between theoretically or abstractly conceived quantities as universals - regularly used in physical laws, without reference to any particular objects possessing and instantiating the quantity in question and concretely understood quantities as particulars, involving specific quantity-instantiating physical objects, events or processes.

\footnotetext{
${ }^{4}$ The reader may be appalled that we are combining two unital length quantities involving different measurement units. This should not be a problem since, for example, in carpentry it is common to combine length values measured in yards with length values counted in inches and fractions thereof. Likewise in physics, length values in centimeters are regularly added to the length values expressed in meters. Concretely, in view of the obvious unitchange conversion rule we have 2 inches +3 millimeters $=53.8$ millimeters $=2.152$ inches, because by definition 1 inch $=25.40$ millimeters and 1 millimeter $=0.04$ inches.
}

Quantities instantiated by classical physical entities together with their values are widely thought to exist independently of whether or not they are ever measured. And we might add that the designation of base quantities is a matter of choice, similar to choosing a coordinate or reference system in space-time models designed for investigating the motions of physical particles and rigid bodies. Simply, there is no intrinsic property of a quantity that makes it fundamental and neither is there any such attribute for measurement units.

Although in the Maxwellian framework the intuitively appealing idea of a physical quantity is not defined and is treated only concretely, on an analogy with the basic operations in the algebra of real numbers there is the often-discussed introduction of quantity calculus, built over the domain of abstractly conceived scalar deterministic physical quantities. In this domain it is customary to stipulate two fundamental algebraic operations:

(i) A commutative and associative operation of addition $Q+$ $Q^{\prime}$ on any pair of quantities $Q$ and $Q^{\prime}$ of the same kind. ${ }^{5}$ In particular, for a coherent pair of units satisfying $[Q]=$ $\left[Q^{\prime}\right]=\left[Q+Q^{\prime}\right]$, the addition is specified by the sum of their numerical values: $Q+Q^{\prime}=\left(\{Q\}+\left\{Q^{\prime}\right\}\right) \cdot[Q]$.

(ii) A commutative and associative product operation $Q \bullet Q^{\prime}$ on any pair of quantities $Q$ and $Q^{\prime}$. In the case of coherent units satisfying $\left[Q \cdot Q^{\prime}\right]=[Q] \bullet\left[Q^{\prime}\right]$, the product is specified by the product of numerical values $\left\{Q \cdot Q^{\prime}\right\}=$ $\{Q\} \cdot\left\{Q^{\prime}\right\}$.

In systematic axiomatic approaches to quantity calculus there is also a multiplication by scalars $\alpha$ having the form $\alpha \cdot Q$, the multiplicative inverse operation $\frac{1}{Q}$, and more generally exponentiation $Q^{n}$ by any integer number $n$. It is a widespread view that with the help of these operations one can define all derived quantities of interest (e.g., mean velocity is defined by the product $\mathcal{L} \bullet \frac{1}{\mathcal{T}}$, where $\mathcal{L}$ denotes the length (distance) quantity and $\frac{1}{\mathcal{T}}$ refers to the multiplicative inverse (quotient) of time quantity).

This may be a rash judgment, however, because (i) besides integer inverses there is also a need for square roots $\sqrt{Q}$ of quantities, (ii) we have to make allowance for variable (e.g., time-dependent) quantities, and (iii) consider differential and integral operators acting on quantities. From the standpoint of abstract algebra, classical quantity calculus looks like a variant of dimensional algebra. Jan de Boer provides more details in [2] and he notes that a full axiomatization of quantity calculus has yet to be completed. In the preceding paragraphs we have already discussed the ratio-based notions of addition and subtraction of quantities. Unfortunately, the definition of product is not this simple. Although we can set

$$
Q \cdot Q^{\prime}=\left(\frac{Q}{Q_{u}} \cdot \frac{Q^{\prime}}{Q_{u^{\prime}}^{\prime}}\right) \cdot Q_{u} \cdot Q_{u^{\prime}}^{\prime},
$$

\footnotetext{
${ }^{5}$ Recall that physical quantities are grouped into disjoint classes of mutually comparable elements.
} 
the real conceptual difficulty is definitional circularity. This problem is cleared up by recognizing that the exact formulation of the notion of product quantities relies on a mathematically deeper idea of symmetric tensor products of onedimensional vector spaces.

Success in developing a powerful theory of quantities depends on well-formed definitions of the notions of quantity, quantity value, and unit. As most metrologists are aware, in the third edition [18] of VIM, henceforth to be referred to as VIM3 (an established acronym for the International Vocabulary of Metrology, derived from its French title), the notions of quantity, quantity value and unit are defined as follows:

Quantity is a property of a phenomenon, body or substance, where the property has a magnitude that can be expressed as a number and a reference.

Quantity value is a number and reference together expressing the magnitude of a quantity.

Unit is a particular quantity, defined and adopted by convention, with which other quantities of the same kind are compared in order to express their magnitudes relative to that quantity.

Lamentably, although there has been significant progress in defining the notion of quantity in a reasonably general and robust way, as evidenced by the regularly updated precisifications of definitions published in the new editions of VIM, there remain difficulties in using these in wider domains of practical applications. Because the intuitively appealing verbal definitions of quantities and related notions tend to employ ordinary language articulations of concepts in a somewhat loose or slippery terminology, there is a large variety of rather different disentangling models of quantities that are compatible with these definitions. Each of the models is furnished with a different structure along with its interpretation and a different degree of empirical adequacy. However, it is important not to be put off by the verbal formulation of definitions of metrological concepts.

As satisfying as our endorsement of verbal formulations might first appear, we must recognize that at least in scientific contexts, the notion of quantity is somewhat technical, depending upon which theory or mathematical model the investigator is considering. For example, the geometric quantities of length and time, the material quantity of mass, and a longer list of derived quantities (velocity, acceleration, kinetic energy, and so forth) are introduced in classical continuum mechanics in a way that is peculiar to that theory. Since it is fully adequate for the purposes of continuum mechanics to treat physical bodies (e.g., rigid materials such as metal rods or beams, discussed in [17]) as continuous substances instead of entities composed of discrete atoms or particles, their unitized length may take any of continuum many possible values. Likewise, in general relativity and quantum mechanics, quantities are appropriately fitted to their specific theoretical contexts. ${ }^{6}$ So a physical quantity is not just a Maxwellian product

\footnotetext{
${ }^{6}$ The most fundamental difference between the classical and relativistic concepts of velicity is seen in their composition. In general relativity, the set
}

factorization into its numerical value and unit, but factorization relative to the quantity's underlying theory or model.

The adequacy of the foregoing definitions of VIM3 remains under heavy debate in metrology. For example, based on these definitions we may be left with the view that there are only directly measured deterministic scalar base quantities. But in applications we encounter derived quantities that are introduced in terms of sophisticated functions of other quantities that are not expressible in an analytically closed form, and of course there are vector and higher-order tensor quantities (e.g., the stress tensor used in continuum mechanics) with matrices of numerical values. Several other important types of quantities also fall outside the scope of the foregoing definitions, including (i) variable quantities that vary with time, space, temperature, and so forth ${ }^{7}$, and (ii) smooth (differentiable) and random quantities that come with particular probability density functions accompanied with measurement units. Evidently, the definitions of VIM3 require further investigation and refinement in order for metrologists to gain a clear and deeper understanding of the all-important concept of quantity, fitted to the context in which their chosen model applies.

\subsection{Conceptual issues in the representationalist founda- tions of measurement}

Perhaps the most elaborate alternative attempt at investigating the nature of quantities, including length, time, mass, velocity and so forth, and their measurement is found in the foundations of measurement, framed and discussed in [13], [15], and [14]. Among social scientists and philosophers of science this approach is widely thought to be the richest storehouse of information on the foundations of deterministic measurement. Unfortunately, because the underlying theory is largely disengaged from much of what is taken to be crucial to natural scientists and engineers in measurement practice, namely the articulation of causal interactions between measured systems and measuring instruments, and the relations between measurement information and measurement uncertainty, it has not received much support among most physicists and engineers, with the possible exception of Finkelstein [6] and those around him.

Here we will give only a brief discussion of the key issues of foundations, referring the reader to the just cited sources for more detail. ${ }^{8}$ In their work, the authors provide an axiomatic basis for measurement, based on so-called representation theorems. Curiously, the co-authors of [13] do not use the term "quantity" at all in any technical sense. Instead, they address the issues of fundamental measurement in terms of suitable attributes of certain classes of objects or events

of values of velocity that exceed the speed of light is empty and is empirically meaningless. In quantum mechanics, time is just a parameter and is not treated operator-theoretically in the same way as the other observable quantities. And so far no final theory of quantum measurement has been articulated and defended.

${ }^{7}$ For example, consider a quantity-theoretic account of the variable height of a large growing tree or the variable mass of a flying aircraft.

${ }^{8}$ However, we shall list a couple of what we take to be good reasons why this approach is fraught with serious conceptual problems. 
having the disposition to instantiate them and possessing a real-valued representing homomorphism. This foundational approach is widely known as the representational theory of measurement.

According to the representational theory of measurement (henceforth acronymed RTM), what is commonly known as base quantities (e.g., mass, length, time and electric current) are treated as homomorphisms from axiomatically specified qualitative empirical order structures ${ }^{9}$ to a quantitative (numerical) structure of the same similarity type, usually given by the familiar naturally ordered additive semigroup structure of positive real numbers. On this approach, to justify the treatment of a given attribute as a basic physical quantity amounts to (i) developing an empirically meaningful axiomatic description of the qualitative order structure characterizing the said attribute, (ii) proving the existence of a homomorphism from this qualitative structure into the order structure of positive reals, and (iii) establishing, when possible, the uniqueness of this homomorphism with respect to some class of transformations, such as (in the case of certain extensive quantities) multiplication by a positive real number.

For some psychologists, RTM offers what they see as an attractive alternative to the traditional so-called classical (or realist as it has been called) definition of measurement, popular in the natural sciences, according to which measurement operations estimate the ratio between the magnitude of a quantity (meaning a quantity as instantiated by some empirical system of interest) and a unit or reference quantity of the same kind. $^{10}$

As alluded to earlier, for a variety of reasons, RTM's approach to quantities and their measurement is fraught with serious conceptual issues. Here we mention only three problems that we regard to be most relevant to the plan of this paper.

Starting with the first problem, from RTM's point of view, what justifies numerical assignments to target systems (i.e., systems instantiating the to-be-measured quantity of interest) is not some physically grounded causal account of interactions between the target system and the quantity's measuring instrument or the system's extant states, but only the fact (established by a representation theorem) that the logicalmathematical structure of the specified qualitative order relations and operations on attribute-bearing systems of interest is faithfully mirrored by the corresponding mathematical relations and operations on numbers assigned to these systems. On this view, quantities are not objective properties of empirical systems in the world, but rather our mathematical representations of designated qualitative attributes of systems. The epistemological significance of such representations is thus

\footnotetext{
${ }^{9}$ These structures consist of a stereotype domain of some attributebearing empirical entities furnished with a qualitative comparison relation and, if applicable, with a qualitative aggregation operation defined on that domain.

${ }^{10}$ The exact numerical value $\{Q\}$ employed in Maxwell's equation may be approximated by a fraction $\frac{n}{m}$ satisfying $m \cdot Q=n \cdot[Q]$ with integer coefficients $m$ and $n$. Clasically, in deterministic situations the estimator $\widehat{Q}$ of the measured quantity $Q$ may be specified by Maxwell's equation of the form $\widehat{Q}=\left\{Q_{ \pm} \mathcal{E}_{Q}\right\} \cdot[Q]$, with maximal interval uncertainty (error) $\mathcal{E}_{Q}$.
}

purely instrumental. Once we establish that "certain aspects of the arithmetic of numbers have the same structure as the empirical situation investigated ... we may then use many of our familiar computational methods of arithmetic to infer facts about the [qualitatively described] empirical structure." This quote comes from [16], page 4, a co-founder of modern RTM. The role of numbers in measurement is thus reduced to serving as convenient computational proxies for qualitative relations among and operations on empirical entities.

In line with its abstract definition of measurement, RTM's treatment of the notion of a unit becomes equally abstract as well. A unit is simply any entity in the domain of a qualitative structure to which a given homomorphism assigns the real number 1 . The issue of objective, publicly accessible instantiations of units becomes conceptually irrelevant. After all, if the only constraint on numerical assignments constituting measurements is logical-mathematical (such assignments must preserve the logical structure of the specified qualitative relations and operations on empirical attribute-bearing systems), then the constraint on the notion of a unit is also purely logical-mathematical, namely that the entity designated as a unit be assigned the real number unit 1 .

Most importantly, the principal selling point of RTM's alternative to the classical definition of measurement has been its repeatedly emphasized bottom-up construction of basic quantities. By claiming that this construction is to be grounded in basic procedures for assigning numbers to objects or events on the basis of qualitative observations of attributes ([13], pp. 1-2), RTM purports to explain and justify without contentious metaphysical commitments or circular theoretical assumptions our passage from simple qualitative observations to quantitative ones ([14], p. 4).

The second problem is RTM's strictly empiricist approach to the foundations of measurement. A close look at RTM's axioms for qualitative structures associated with measurable attributes reveals that these axioms invariably transcend simple qualitative observations and posit hypothetical infinite precisions that exceed the capacities of human measurement. (For some additional details and discussion of related issues, see [4] and [5].)

Although there are many philosophical discussions of interpretive difficulties of RTM, here we mention just one, namely a principal obstruction to RTM's attempt to ground measurement of basic quantities in qualitative observations and manipulations of observable empirical entities. The roots of the problem lie in the assumptions of representation theorems. It is presumed that the equivalence relation on objects with respect to their manifestations of a given attribute is a mathematical congruence relation, whereas the relation induced by measurement-based qualitative comparison judgments is only an empirically determined relation of indistinguishability. By endowing indistinguishability with the (structurally strictly richer) logical properties of congruence, RTM ends up requiring observation-based qualitative comparison judgments to have unlimited arbitrarily perfect accuracies. This requirement obviously transcends the necessarily finite discrimination capacities of any physically realiz- 
able measurement device, let alone of human sensory organs responsible for "simple qualitative observations." As a result, RTM's claim about the empirical significance of its definition of quantities as structure-preserving mappings from qualitative structures into real numbers becomes unpalatable. In view of these highly idealizing assumptions we are left without empirically meaningful interpretations of qualitative structures.

Finally, the third problem concerns RTM's instrumentalist conception of measurement as assignments of numbers to things in the world. This conception comes to grief when combined with RTM's formal treatment of quantities in terms of real-valued homomorphisms. The reason is that almost all real numbers are (in the increasing order of complexity) irrational, transcendental, non-computable, and random. Not only do such numbers embody an infinite amount of information, this information (in the case of the last two kinds) cannot be obtained with finitary information-processing resources even in the limit of infinite time. Yet RTM's representation theorems allow real-valued homomorphisms to take as values arbitrary real numbers, which means that measurement (qua assignment of numbers) requires omniscient measuring agents with unlimited capacities, allowing them to obtain and manipulate infinite amounts of non-computable or random information. Once again, the empirical significance of RTM's homomorphism-based definition of quantities becomes questionable in light of the fact that real-world scientists perform measurements without having superhuman capacities required by RTM.

These three problems point to what we see as a fundamental flaw in RTM. Namely, it conflates the radically different epistemological and ontological sides of measurement operations. On the ontological side of measurement, our accepted scientific theories tell us which quantitative attributes are possessed by natural systems in the world (e.g., the class of attributes of a rigid steel beam typically includes length, mass, and temperature, to mention just some). And if our accepted theories describe temporal changes in the values of quantities by differential equations over real-valued functions, then the ontological features they impute to the natural world include continuous changes in the amounts of attributes possessed by natural systems. This, in turn, means that, as a rule, the exact values of these attributes are in principle inaccessible to finite measuring agents (e.g., the exact numerical value of the steel beam's length may be a non-computable real number). In short, the ontological claim that some attributes of natural systems in the world are continuous (real- or complex-valued) quantities is a fundamental theoretical postulate of science which can only be confirmed but never completely verified by finite and discrete amounts of information obtainable by human agents.

The epistemological side of measurement, by contrast, is determined by the ways in which measuring agents can obtain information about the values of quantitative attributes possessed by natural systems. The fundamental epistemological fact of measurement is that information obtainable by measuring agents is always finite and discrete, making it in prin- ciple impossible for them to know the exact values of continuous quantities. In light of this fact, it is paramount for a theory of measurement to provide a formally precise and empirically meaningful account of (i) how the structurally impoverished (finite and discrete) epistemological side of measurement relates to the structurally rich ontology of continuous quantities postulated by and operative in science, and (ii) how this relation allows measuring agents to obtain mathematically tractable and scientifically useful estimates of continuous quantities characterizing the natural systems of interest.

With this distinction in mind, it is now easy to see how RTM conflates the epistemology and the ontology of measurement by forcing both into a single model it calls qualitative structure. For example, the binary relation "shorter than" considered in the domain of rigid metal beams is given an epistemological interpretation in terms of qualitative comparison judgments. Yet, in order to capture enough of the much richer ontological structure of quantitative attributes required for their real-valued representation, the stipulated logicalmathematical properties of this relation must transcend the epistemological limits of finite measuring agents. Similarly, the familiar concatenation operation introduced in the domain of rigid metal rods is given an epistemological interpretation in terms of some qualitative manipulations of empirical entities, but the stipulated logical properties of this operation required for real-valued representations (e.g., the algebraic closure property implying arbitrarily many iterations of concatenation) take it beyond the empirically meaningful limits of what can be achieved by finite measuring agents. ${ }^{11}$ The cost of RTM's conflation of epistemology and ontology, as we have already noted, is an irreconcilable conflict between empirical interpretability and numerical representability of RTM's qualitative structures.

The upshot of the present discussion is that qualitative measurement structures which support representation theorems are not empirically interpretable (which deprives RTM's representation theorems of their alleged empirical significance), while empirically interpretable qualitative structures do not support representation theorems (which deprives RTM of its principal theoretical achievement).

The preceding discussion of these problems along with the criticisms raised earlier are sufficient, we believe, to convince the reader that RTM is not well connected with our understanding of physical measurement and is too flawed to be a viable alternative to the classical realist approach.

What the current classical approach lacks at present, however, is a mathematically rigorous analysis of the foundational aspects of measurement that upholds the pragmatically and conceptually motivated objections to RTM canvassed earlier,

\footnotetext{
${ }^{11}$ To simplify our analysis, we sidestep a longer list of other obvious requirements. For example, it is tacitly assumed that each metal beam is neither extremely long, hot or heavy, nor too short, cold or of little weight, so that it is physically manipulable within the bounds of normal human abilities. For another example, the process of determining the beam's exact length makes no sense without first establishing its precise left and right spatio-temporally co-localized endpoints.
} 
and definiteness in serving as an intuitively appealing, practically useful, robust and mathematically attractive framework for quantities and their measurement. In what follows we will present a characterization of quantities that is able to handle both deterministic and probabilistic quantity calculus and quantity measurement.

\subsection{Towards a state-based ontology of unital quantities}

Modern treatments of deterministic quantities skirt the issue of truth-conditions for metrological assertions. Based on what we said earlier, it is apparent that RTM replaces the idea of truth conditions with that of a 'qualitative structure.'

To fix ideas, it is useful to have a simple example in mind. Consider the following question: what exactly is it about the flagpole $\mathfrak{f}$ under consideration (as a material body treated in continuum mechanics) in virtue of which the metrological statement, say, " $f$ is 5.4 meters long" is true? In other words, the question is about something which directly pertains to the flagpole's extant physical mode of being and which serves as the truth-maker of statements about its length. ${ }^{12}$

On the representational approach to quantities, the truthmaking feature of flagpole $\mathfrak{f}$ is understood in an essentially structuralist way. As mentioned above, what makes the statement " $f$ is 5.4 meters long" true is not something intrinsic to $\mathfrak{f}$, but only that (i) $\mathfrak{f}$ belongs to a certain structured domain of length-bearing bodies (RTM's "qualitative structure"), and (ii) there is a structure-preserving real-valued function on that domain which assigns number 1 to physical bodies designated as "prototypes" for the meter unit of measure, and real number 5.4 to the flagpole $\mathfrak{f}$.

We find this account of corroborating the validity of metrological statements to be unsatisfactory even if we set aside RTM's empiricist assumption that the structured domain in question is definable on the basis of simple qualitative manipulations of observable entities. The stipulated structural features of the said domain (required for the representation theorem) prevent us from interpreting its elements even as just actual length-bearing objects (whether observable or not), if only because the actual world does not contain objects produced by arbitrarily iterated compositions of flagpoles, beams, etc., not to mention their arbitrarily iterated compositions with themselves. Also, the relevant relations among actual length-instantiating objects are not immune to the effects of thermal expansion, corrosion, humidity and other real-world factors affecting the actual flagpoles, metal rods, and so forth. Perhaps the elements of this structured domain can be interpreted as possible flagpoles, possible beams, and

\footnotetext{
${ }^{12}$ The fact that certain rigid material bodies are chosen as the preferred referents of our discourse about length does not mean that we turn our back on the underlying quantum mechanical structure of matter. Even though we hold quantum mechanical principles to be fundamental, there are many effective ways of modeling quantities and units in the home language of continuum mechanics and electromagnetism. Thus, setting aside quantum physics considerations is not just a matter of idealization, but an established practice of metrological science we follow. In particular, as seen in [10], it is an accepted standard to conform to definitions of quantities, units and related measurement-theoretic concepts common within the traditional frameworks of physical sciences.
}

so on, but even then the above explanation will remain unsatisfactory, due to the needless obscurity of its modal or counterfactual background metaphysics.

Earlier we argued that the traditional realist approach to quantities is to be preferred over RTM, in view of its most hospitable environment for deterministic quantity calculus. But curiously, this framework seems to be not much better off than RTM in regards to truth semantics. It is simple enough to see that the standard classical approach to quantities (discussed in Section 1.1) is axiomatically built and is strictly syntactic in nature. From a semantic standpoint, more needs to be said by the classical theory about exactly how a valid metrological assertion about a quantity of interest is obtained. We shall now enter into the details of how are we to reason about the truth conditions of metrological statements.

With classical continuum mechanics as our background theory, a metaphysically modest alternative to RTM's approach to truth conditions is to accept that (i) physical objects possess a well-defined state at any given moment of time, and (ii) there are deterministic causal laws that characterize the temporal evolution of these states. If we have these conditions, then what matters about the flagpole $f$ in the context of predicting and/or measuring its length is that it is in a certain physical-geometric condition (that may change over time, e.g., due to thermal expansion).

We can now put our conceptual pieces together. The notion of state has its home in classical systems theory. However, the standard state space modeling practice embraces a far more generous state ontology than required by quantity calculus. On this classical well-established view, at any instant of time a target system's state is assumed to provide a complete description of the system's physical mode of existence. Thus, knowing the system's state implies knowing everything that is necessary to predict the future of that system. We differ from this complete state approach in considering only certain incomplete quantity-restricted states that are fully sufficient for serving as truth-makers of metrological claims about the values of the quantity under consideration. ${ }^{13}$ Our goal is to provide a state space description of systems that captures everything that is necessary and sufficient for a complete specification of values of the system's quantity of interest.

In the example of length we know that at any moment of time the flagpole $\mathfrak{f}$ occupies a specific spatial region in the common-sense Newtonian space and time with two spatial endpoints that determine a unique connecting line segment.

\footnotetext{
${ }^{13}$ Such incomplete states are quite common in the natural sciences. For example, in the Lotka-Volterra model of population growth it is customary to specify biological states only in terms of population sizes of predators and preys, even though biological populations are known to be blessed with many other biologically essential attributes. Hidden variable theories of quantum systems are also based on the notion of incomplete states. One might object that the assumption of incomplete states is tantamount to admitting that a complete description exists or perhaps might yet be found. Yes, of course, but notice that in all this it is implicitly assumed that we are considering an entire algebra of quantities (discussed in detail, e.g., in [4]) that exhaustively specifies all of the system's properties at a given time. However, when we are not interested in the fullest description of the target system, but only in a particular aspect of it, specified by a quantity of interest, then even incomplete states are sufficient to determine its values.
} 
And the Euclidean length of this line segment defines the flagpole's length in a chosen unit. Thus, in the case of length quantity applied to the target flagpole we may specify its state space $\mathscr{S}_{\uparrow}$ by the set of all line segments, modulo spatial congruence. We take it as our fundamental assumption that by instantiating a line segment, this state space links $\mathfrak{f}$ to a wellunderstood and much studied framework of affine Euclidean geometry. The instantiation relation between length-bearing material bodies (like flagpoles and beams) and line segments allows us to consider also empirical comparison relations between and concatenation operations on certain length-bearing bodies, thought of as instantiating the familiar "shorter than" relation between and the composition operation on the corresponding line segments. For these additional reasons, the flagpole's length-constrained state space of line segments is furnished with a surplus structure of an ordered semigroup $\left\langle\mathscr{S}_{\uparrow}, \prec, \boldsymbol{\Psi}\right\rangle$, to be discussed in the next section.

To get clear on the connection between quantities and units, it would be good first to have some idea of what quantities and units really are. For a long time it has been clear that it is fruitful to define quantities in terms of real-valued functions on stereotype domains of physical objects or events (see [7] for a semiformal and philosophical discussion). Unfortunately, such functions come in a great variety of types: continuous and discrete, deterministic and random, ordinary and structure-preserving, and so on. We know that RTM finds the measurement-theoretic fruitfulness of such functionbased treatments of quantities solely in terms of real-valued homomorphisms between relational structures.

In contrast to RTM, in our framework the abstractly conceived deterministic unital quantities are modeled by positive real-valued isomorphisms between a physical-geometric state space and the space of positive real numbers. For instance, a concrete unital length quantity of flagpole $\mathfrak{f}$ is given by an isomorphism function $\mathcal{L}_{\mathfrak{f}}: \mathscr{S}_{\uparrow} \longrightarrow \mathbb{R}_{+}$between the flagpole's state space and positive reals. Why isomorphism? Because, as we will explain at the top of the next section, under isomorphism unital quantities and units encoded by states stand in a mathematical relationship of duality. And, importantly, this in itself turns out to be extremely important in quantity calculus. In a bit more detail, here the idea of duality refers to a one-to-one correspondence between quantities and states of a system under consideration. As we shall see below, the fact that quantities and states can be formulated in terms of a dual relationship is crucial for a formal justification of merging units and quantities into a single notion of a unital quantity. We should note that it is dimension-theoretically profitable to view the collection of all unital quantities of a given kind as their quantity type. The upshot is that we can provide a formal framework in which the idea of a physical-geometric attribute, e.g. length, is formally accommodated by the type of all unital lengths.

Unital quantities have two salient features: (i) there is a one-to-one and onto correspondence between unital quantities and their units, rigorously characterized by states, and (ii) the unit of the unital quantity $Q$ of interest is uniquely specified by the state $u=Q^{-1}(1)$ to which the quantity assigns the unit real number 1 , i.e., the identity condition $Q(u)=1$ holds. ${ }^{14}$

So now we can make the claim about the flagpole's length mathematically explicit and rigorous as follows:

Length $(\mathfrak{f})=\mathcal{L}_{\mathfrak{f}}\left(\mathrm{AB}_{\mathfrak{f}}\right)=$ Euclidean distance-in-meters $(\mathrm{A}, \mathrm{B})$,

where $\mathrm{AB}_{f}$ denotes the line segment with endpoints $\mathrm{A}$ and $\mathrm{B}$, realized by the flagpole's physical-geometric state and the Euclidean distance in meter measurement unit between them is a positive real number.

Thus, the assertion " $f$ is 5.4 meters long" is true if and only if the following three non-epistemic conditions hold:

(i) The flagpole's extant physical-geometric state instantiates a unique line segment $\mathrm{AB}_{\mathfrak{f}}$ in $\mathscr{S}_{\mathfrak{f}}$;

(ii) The physical-geometric state of a physical body designated as a prototype meter instantiates a unique line segment $s_{m}$ such that its unitized length $\mathcal{L}_{\mathfrak{f}}$ satisfies the unitizing condition $\mathcal{L}_{\mathfrak{f}}\left(\mathrm{s}_{\mathrm{m}}\right)=1$, and

(iii) the unital length unitized by $\mathrm{s}_{\mathrm{m}}$ satisfies the assertion's condition $\mathcal{L}_{\mathfrak{f}}\left(\mathrm{AB}_{\mathfrak{f}}\right)=5.4$.

Note that these conditions hold (or fail to hold) regardless of whether $\mathfrak{f}$ is ever subjected to any kind of measurement. When we do measure the flagpole's particular unital length (in this case length-in-meters), our aim is to estimate the unknown numerical value of this unital length. The crucial point here is that this unknown numerical value is an objective mathematical property of a certain line segment, and the flagpole's physical-geometric state inherits this mathematical property by instantiating that segment. We regard this to be the underlying fundamental ontological assumption about the truth-making role of measured systems. Thus, if we estimate the value of $\mathfrak{f}$ 's unital length as, say, lying in the half-open interval $[5.35,5.45)$ of meters, the measurement output gives us information about an objective numerical property inherited by (and therefore characteristic of) $\mathfrak{f}$ 's physical-geometric state.

Because the details of truth conditions are placed where they belong (they are fitted to the investigated unital quantity's theoretical context), as may be expected, the state spaces for different quantities, such as mass and electric current, will be different. For example, combination of multiple quantities instantiated by a complex system leads to a product state space. To avoid certain distracting complications, we cast the definitions of units in terms of (pure) deterministic states. However, we shall later consider also statistical states, encoded by certain probability density functions on the underlying space of pure states.

Let us be clear about how units of length enter into the state space framework. As well-known, since 1983 the meter

\footnotetext{
${ }^{14}$ Note that we are making a sharp distinction between units specified by designated states and carefully prepared physical prototypes or etalons instantiating these units, characterized by states. Although in this setting there are continuum many possible units, in practice only a relatively small number is sufficient, usually a decimal multiple or decimal fraction of a designated base unit.
} 
unit is defined (as displayed on page 18 in [10]) somewhat theoretically in terms of an Euclidean spatial line segment in the following way:

One meter is the length of a straight-line path traveled by light in a vacuum during the time interval of $1: 299,792,458^{\text {th }}$ of a second.

So now because 1 meter is officially defined as the length of a designated straight-line segment, we have a good reason for sticking to a line-segment representation of states for length quantities.

To ground our intuitions on how quantity-constrained states work and what makes a unit of measure, consider another simple and very important example of an extensive quantity, namely electric current. To that end, we recall the SI definition of the ampere (= amp) base unit of measure for electric current:

One ampere is equal to the constant current which, if maintained in two straight parallel conductors of infinite length, of negligible circular cross-section, and placed 1 meter apart in a vacuum, would produce between these conductors a force equal to $2 \times 10^{-7}$ Newton per meter of length ([10], p. 113).

Before continuing with the introduction of currentconstrained states of powered closed-loop electric circuits, three comments are called for.

First, note that the advocates of RTM would find it impossible to express the foregoing counterfactual definition of the ampere unit in the framework of qualitatively assertible facts, encoded by RTM's empirical structures. Clearly, to deal with "parallel conductors of infinite length" would exceed the capacities of human measurement.

Note also that the definition relies on Ampére's force law of classical electrodynamics that employs the derived quantity of force per length unit and it does not take any cues from the ontology of quantum theory, as evidenced by the conductors' characterization of having "negligible circular cross-section." In contrast, metrologists working with the SI framework for measurement units find these theory-anchored non-prototypebased definitions to be fundamental in the development of metrological science. But of course, not everyone is pleased by the use of derived quantities (e.g., force) in the definitions of base units.

Second, it is common knowledge that in applications, electric current is realized by a closed-loop electric circuit (powered with a current source, e.g., a battery or generator) that can be set up in many ways, usually schematized (under idealizing assumptions) by a diagram of electrical connections between basic electrical components.

The simplest nontrivial electric circuit consists of an electrical energy source, an energy-consuming load (e.g., a resistor) and a control device (e.g., a switch), all connected serially by conducting wires. The electrical behaviors of circuits are established on the basis of values of currents and voltages in the given circuit. ${ }^{15}$
Since in parallel combinations of circuits there are multiple paths by which the electric current can flow, the total amount of current passing through the parallel circuit is equal to the sum of current values in the individual branches of the circuit. Here the upshot is the inherent ordered additive semigroup structure of currents in parallel circuits (the voltage drop is the same across each branch of the circuit).

Third and final, suppose we are given a well-powered closed-loop parallel electric circuit $c$ and are interested in the total amount of current that flows through all of its branches. Let the metrological statement about the current's value be " $I(\mathfrak{c})=2.5$ amps." Once again, we might ask: what is the truth-maker of this statement? As a related issue, how would one test the validity of this statement? The intuitive answer is: the statement is true provided that there is a specific electrical energy-transporting activity going on in the powered parallel circuit that matches the stated value of amps. So the perplexity is resolved by attributing a certain electrical state directly to the circuit.

We can go down the road of quantum physics and give a rigorous characterization of truth-makers of metrological statements about electric currents in terms of flows of electrically charged particles that can be counted. Concretely, since one ampere is known to be equivalent to one coulomb of charge passing past a point of $c$ in one second, meaning the flow of approximately $\mathbf{i}=6.24 \times 10^{18}$ electrons per second in a powered circuit, we now have a direct empirical support for the validity of the claim " $\mathcal{I}(\mathfrak{c})=2.5$ amps." Namely, the sentence is true just in case approximately $2.5 \times \mathbf{i}$ electrons pass through a cross section of c's conducting wire per second.

Surprisingly, this recipe for testing the truth value of electrical statements seems to have a major flaw in that it applies only to currents specifiable by the number of free electrons flowing from the positive to the negative terminal of c's power source per second.

In contrast to quantum interpretation, one important lesson we have learned earlier is that we cannot decouple the definition of classical quantities and their units from the theory that uses them in its laws and measurement. In particular, observe that the now-preferred definition of ampere makes no reference at all to the details of moving electric charges of particles. Instead, it is deeply connected with the classical theory of electrodynamics, in which a unitized electric current may take any positive real value whatsoever. So like it or not, we want to fasten on the idea of classical electrodynamics in which electric current is deliberately idealized as a kind of substance that moves continuously through a circuit wire. Upon switching between quantum and classical viewpoints, current can be seen in a perfectly legitimate manner as an instantaneous rate of electrical charge movement, stipulated by the familiar differential equation $\mathcal{I}=\frac{\mathrm{d} Q}{\mathrm{dt}}$ that conveniently idealizes away the discrete and finitary character of particle charges.

\footnotetext{
${ }^{15}$ For ease of exposition, we are restricting our attention to human-made circuits and put aside the technical nuances of electric currents in electrolytes, sparks, lightnings, solar winds, and currents in the ambience of changing magnetic fields.
} 
So a more promising route is to specify the state space $\mathscr{I}_{c}$ of circuit $c$ by the set of all electrical currents, maintained in any two straight parallel conductors, as explicated in the definition of ampere. Depending on the strength of generated forces, the currents may be weaker or stronger and as we mentioned earlier, they compose additively in parallel circuits. Our main point is that, once again, we can use the ordered semigroup of the form $\left\langle\mathscr{I}_{c}, \prec, \boldsymbol{\Psi}\right\rangle$ as a state space structure of the electric circuit $c$. This structure, in turn, allows to define unital current quantities in terms of isomorphisms of the form $\mathcal{I}_{c}: \mathscr{I}_{\mathrm{c}} \longrightarrow \mathbb{R}_{+}$between the circuit's state space and positive reals. And as we saw earlier, a unital current quantity $\mathcal{I}$ is unitized by the unique electric state $\mathrm{i}_{\mathrm{amp}}$ provided that $\mathcal{I}\left(\mathrm{i}_{\mathrm{amp}}\right)=1$.

In the present deterministic quantity-theoretic framework the assertion "Current-in-amps in circuit $c=2.5$ " is true just in case the following three conditions are satisfied:

(i) The net movement of charged particles in the powered circuit $c$ instantiates a unique electrical state $\mathrm{i} \in \mathscr{I}_{i}$;

(ii) Motion of charged particles in a "prototype circuit" realizing the conditions of definition of 1 ampere instantiates the designated electrical state $\mathrm{i}_{\mathrm{amp}}$ such that the circuit's unitized electric current $I_{\mathrm{c}}$ satisfies the unitizing condition $\mathcal{I}_{\mathrm{c}}\left(\mathrm{i}_{\mathrm{amp}}\right)=1$, and

(iii) the unital current unitized by i imp satisfies the assertion's condition $\mathcal{I}_{\mathrm{c}}(\mathrm{i})=2.5$.

Although for the large part of this introduction we have investigated by way of examples only the respective state space structures of unital length and unital electric current, the proposed approach applies equally well to the other gardenvariety of extensive quantities. To summarize, as we have argued, neither RTM nor the often cited Maxwellian approach solves the problem of units and truth conditions for metrological statements. We have identified two main obstacles that are in the way of handling these issues: (i) missing state space, and (ii) the missing isomorphism condition. It is now time to explain the algebraic requirements for unitized quantities.

\section{UNITAL QUANTITY CALCULUS AND QUANTITY TYPES}

We have just seen that it makes a lot of empirical sense to describe the quantity-restricted states of systems, instantiating a quantity of interest, by the elements of an ordered semigroup. However, to be able to use this algebraic tool kit effectively, we need to model unital quantities as isomorphisms between the ordered semigroups of states and that of positive real numbers. The great power of the dual connection between states and unital quantities shows up in solving the earlier mentioned problems pertaining to the truth conditions of measurement statements and relations between quantities and units.

\subsection{States and unital quantity calculus}

Among all the algebraic ingredients that go into building a unital quantity calculus, two stand out as especially fundamental: (i) complete ordered semigroups of states, and (ii) semilinear spaces of compatible unital quantities.
Why complete ordered semigroups and not just ordered semigroups? Because in general there is no reason to think that there are any isomorphisms between ordered semigroups and the semigroup of positive real numbers. However, we know from the seminal work of Hölder [8] that complete ordered semigroups uphold such isomorphisms. So in our framework a complete ordered semigroup is viewed as a fixed universal conceptual backdrop against which quantity calculus unfolds. Here is the definition:

Definition 1: A complete ordered semigroup $\langle\mathfrak{G},<,+\rangle$ is a set $\boldsymbol{S}$ equipped with a strict total order relation $<$ and a binary associative operation + such that the following conditions are satisfied:

(i) The order structure $\langle\boldsymbol{G},<\rangle$ is a totally ordered set.

(ii) The algebraic structure $\langle\boldsymbol{G},+\rangle$ is a commutative and cancellative semigroup, i.e., for all $\mathfrak{a}, \mathfrak{b}$ and $\mathfrak{c}$ in $\mathfrak{\subseteq}$ we have

$$
\mathfrak{a}+\mathfrak{b}=\mathfrak{b}+\mathfrak{a},
$$

and

$$
\mathfrak{a}+\mathfrak{c}=\mathfrak{b}+\mathfrak{c} \Longrightarrow \mathfrak{a}=\mathfrak{b}
$$

(iii) The monotonicity

$$
\mathfrak{a}<\mathfrak{b} \Longrightarrow \mathfrak{a}+\mathfrak{c}<\mathfrak{b}+\mathfrak{c}
$$

together with strict positivity

$$
\mathfrak{a}<\mathfrak{a}+\mathfrak{b}
$$

and the solvability

$$
\mathfrak{a}<\mathfrak{b} \Longleftrightarrow \mathfrak{a}+\mathfrak{c}=\mathfrak{b} \text { for some } \mathfrak{c}
$$

conditions hold for all $\mathfrak{a}, \mathfrak{b}$ and $\mathfrak{c}$ in $\mathfrak{G}$.

(iv) Every upper-bounded nonempty subset $\left\{\mathfrak{a}_{i} \mid i \in I\right\} \subset \boldsymbol{\Im}$ has a supremum, denoted $\bigvee_{i \in I} \mathfrak{a}_{i}$, such that for all $\mathfrak{b}$ the following general distributive law

$$
\mathfrak{b}+\bigvee_{i \in I} \mathfrak{a}_{i}=\bigvee_{i \in I}\left(\mathfrak{b}+\mathfrak{a}_{i}\right)
$$

holds.

Complete ordered semigroups come with a natural order topology. A subbase of this topology consists of all subsets of the form $\{\mathfrak{b} \mid \mathfrak{b}<\mathfrak{a}\}$ or $\{\mathfrak{b} \mid \mathfrak{a}<\mathfrak{b}\}$ for some $\mathfrak{a}$. Based on topology, these semigroups can also be viewed as Borel measurable spaces, required for a precise definition of random unital quantities and their probability density functions.

The next thing we must do is specify what counts as an effective mapping between pairs of complete ordered semigroups. As we know, in representational measurement theory of particular importance are certain embedding maps from ordered semigroups to the real line. In more detail, the following notion is deliberately fit for measurement applications.

If $\langle\boldsymbol{\Xi},<,+\rangle$ and $\left\langle\boldsymbol{\Xi}^{\prime},<,+\right\rangle$ are complete ordered semigroups, a mapping $\mathcal{F}: \mathfrak{S} \longrightarrow \mathfrak{S}^{\prime}$ is called a complete ordered semigroup embedding when 
(i) $\mathfrak{a}<\mathfrak{b} \Longleftrightarrow \mathcal{F}(\mathfrak{a})<\mathcal{F}(\mathfrak{b})$,

(ii) $\mathcal{F}(\mathfrak{a}+\mathfrak{b})=\mathcal{F}(\mathfrak{a})+\mathcal{F}(\mathfrak{b})$, and

(iii) $\mathcal{F}\left(\bigvee_{i \in I} \mathfrak{a}_{i}\right)=\bigvee_{i \in I} \mathcal{F}\left(\mathfrak{a}_{i}\right)$.

hold for all $\mathfrak{a}, \mathfrak{b}$ and $\mathfrak{a}_{i}$ in $\mathfrak{S}$. If in addition to the embedding property, $\mathcal{F}$ maps $\mathfrak{S}$ onto $\mathfrak{S}^{\prime}$, then we say that the two complete ordered semigroups are isomorphic and write $\mathfrak{S} \cong \mathfrak{S}^{\prime}$. Reasoning along these lines will convince the reader that unital quantities are best thought of as isomorphisms between a complete ordered semigroup of states of a given kind and the complete ordered semigroup of positive reals.

Of special interest is the relationship between two unital quantities of the same kind. In complete analogy with geometric coordinate changes, conversion of a given unital quantity $Q$ into another unital quantity is achieved by composing $Q$ with a suitable similarity automorphism $1_{\alpha}$, as shown in the commutative diagram

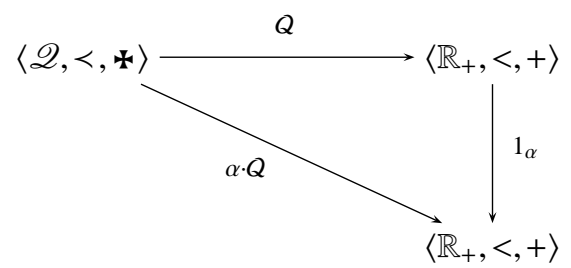

in which we have the equality $\alpha \cdot Q=1_{\alpha} \circ Q$. We hasten to add that for any conversion coefficient $\alpha>0$ the map $1_{\alpha}$ is a complete ordered semigroup isomorphism (in this case an automorphism), defined by $\left[1_{\alpha}\right](1)={ }_{d f} \alpha$ and $\left[1_{\alpha}\right](\beta)={ }_{d f} \alpha \cdot \beta$ for all $\beta>0$. Thus, all unital quantities are of the form $\alpha \cdot Q$, where $Q: \mathscr{Q} \longrightarrow \mathbb{R}_{+}$is a designated reference unital quantity and $\alpha>0$ is a conversion coefficient in $\mathbb{R}_{+}$. It is an elementary exercise to show that the automorphism group $\operatorname{Aut}\left(\left\langle\mathbb{R}_{+},<,+\right\rangle\right)$ of conversion coefficients (automorphisms) is isomorphic to the ordered multiplicative group of strictly positive reals.

In a dual manner, we can also consider dynamical automorphisms of the form

$$
\langle\mathscr{Q},<, \boldsymbol{\Psi}\rangle \stackrel{F}{\longrightarrow}\langle\mathscr{Q}, \prec, \boldsymbol{*}\rangle
$$

that transform given states to new states (i.e., given measurement units to new units). For example, the physicalgeometric state of the flagpole under consideration smoothly changes with smooth (heating or cooling) variations of the ambient temperature. This brings out an important one-toone quantity-state duality relationship for any pair $Q, Q^{\prime}$ : $\mathscr{Q} \longrightarrow \mathbb{R}_{+}$of unital quantities: the conversion automorphism $Q^{\prime} \circ Q^{-1}: \mathbb{R}_{+} \longrightarrow \mathbb{R}_{+}$is in a one-to-one correspondence with the state change automorphism $Q^{-1} \circ Q^{\prime}: \mathscr{Q} \longrightarrow \mathscr{Q}$ that can be thought of as a typical example of $F$.

At this stage we slip in a remark about the length of two juxtaposed, i.e., concatenated flagpoles, symbolized $\mathfrak{f}+\mathfrak{f}^{\prime}$. Since each flapole comes with its own complete ordered semigroup of physical-geometric states encoded by line segments, in the simplest situation of two juxtaposed flagpoles their states are represented by the product space $\mathscr{S}_{i+\uparrow^{\prime}}=d f \mathscr{S}_{\uparrow} \times \mathscr{S}_{\dagger^{\prime}}$ of identical factor state spaces and the product $\mathcal{L}_{\mathfrak{f}} \times \mathcal{L}_{\mathfrak{f}^{\prime}}$ of constituent unital quantities. These quantities are transformed into their addition using the addition operation on reals. Concretely, as shown in the commutative diagram

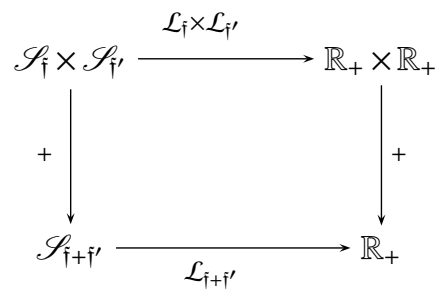

the total unital length is given by the additive composite $Q_{\mathfrak{f}+\mathfrak{f}^{\prime}}\left(\mathrm{s}+\mathrm{s}^{\prime}\right)=_{d f} \mathcal{L}_{\mathfrak{f}}(\mathrm{s})+\mathcal{L}_{\mathfrak{f}^{\prime}}\left(\mathbf{s}^{\prime}\right)$ for all states $\mathrm{s}$ and $\mathrm{s}^{\prime}$ instantiated by the respective flagpoles $\mathfrak{f}$ and $\mathfrak{f}^{\prime}$. This algebraic apparatus immediately generalizes to other finitely many juxtaposed flagpoles, and in fact to any finite collection of lengthbearing physical objects.

It is noteworthy that the foregoing product construction works equally well also for parallel combinations of electric circuits in which currents are known to behave additively: $I_{\mathrm{c} \| \mathrm{c}^{\prime}}=\mathcal{I}_{\mathrm{c}}+\mathcal{I}_{\mathrm{c}^{\prime}}$. Actually, the above product state space method is very general; it works for all kinds of unital quantities. For example, the unital area of a rectangle instantiated, say, by a hallway $\mathfrak{h}$ of interest, is determined by the product of the unital lengths of its sides: $\mathcal{A}_{\mathfrak{h}}\left(\mathrm{s}, \mathrm{s}^{\prime}\right)=\mathcal{L}_{1}(\mathrm{~s}) \cdot \mathcal{L}_{2}\left(\mathrm{~s}^{\prime}\right)$. We can express this derived geometric quantity by the commutative diagram

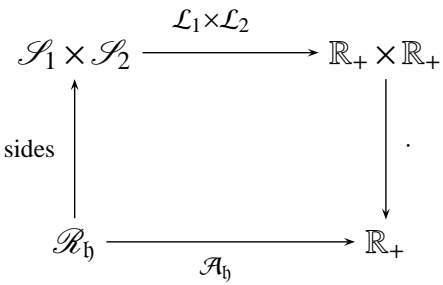

in which the space $\mathscr{R}_{\mathfrak{h}}$ encodes the possible physicalgeometric states of the hallway $\mathfrak{h}$.

Finally, here is a very important example of a temporally varying unital length quantity $\mathcal{L}_{\text {tr }}(\mathrm{t}): \mathscr{S}_{\text {tr }} \longrightarrow \mathbb{R}_{+}$, instantiated by the variable height of, for example, a large growing tree tr, considered at time instant $t$. As might have already become clear, the state space $\mathscr{S}_{\text {tr }}$ plays two essential roles: (i) a synchronic role in the quantity-constrained specification of the system's mode of being at a particular time, sufficient for the determination of the target system's quantity values of interest, and (ii) a diachronic role in modeling the temporal evolution of the tree's stages of growth.

To model the tree's temporal dynamics of states, we will need a totally ordered space $\langle\boldsymbol{T}, 0,<\rangle$ of positive time instants, starting with the initial moment of time 0 , and a dynamical map $\mathcal{D}_{\mathrm{t}}: \mathscr{S}_{\text {tr }} \longrightarrow \mathscr{S}_{\text {tr }}$ with $\mathrm{t} \in \boldsymbol{T}$ that completely characterizes the deterministic change of the tree's height. Suppose the tree's state (i.e., its instantiating height, encoded by a particular line segment) $s_{t}$ at time $t$ is specified by the dynamical law $\mathrm{s}_{\mathrm{t}}=\mathcal{D}_{\mathrm{t}}\left(\mathrm{s}_{0}\right)$, where $\mathrm{s}_{0}$ captures the state considered at time 
zero, for instance, at the time when the tree was planted. Under temporal precedence $t<t^{\prime}$ we can see that the following condition holds:

$$
\mathcal{L}_{\mathrm{tr}}\left(\mathrm{s}_{\mathrm{t}^{\prime}} \dot{-} \mathrm{s}_{\mathrm{t}}\right)=\mathcal{L}_{\mathrm{tr}}\left(\mathrm{s}_{\mathrm{t}^{\prime}}\right) \dot{-} \mathcal{L}_{\mathrm{tr}}\left(\mathrm{s}_{\mathrm{t}}\right)
$$

where the subtraction operations meet all the conditions of meaningfulness. Upon defining $\mathcal{L}_{\mathrm{tr}}(\mathrm{t})={ }_{d f} \mathcal{L}_{\mathrm{tr}} \circ \mathcal{D}_{\mathrm{t}}$, so that the equality $\left[\mathcal{L}_{\mathrm{tr}}(\mathrm{t})\right](\mathrm{s})=\mathcal{L}_{\mathrm{tr}}\left(\mathrm{s}_{\mathrm{t}}\right)$ holds, we make the idea of a time-dependent unital height fully precise by the commutative diagram

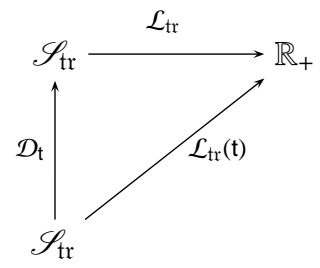

In the simplest case of linear deterministic growth we can set

$$
\mathcal{L}_{\mathrm{tr}}\left(\mathrm{s}_{\mathrm{t}^{\prime}} \dot{-} \mathrm{s}_{\mathrm{t}}\right)=\boldsymbol{\alpha} \cdot\left(\mathrm{t}^{\prime} \dot{-} \mathrm{t}\right),
$$

where the measurement unit of the coefficient of growth $\alpha$ must be fitted to the equation's context, i.e., it must be an appropriate length unit over a time unit.

This approach to variable unital quantities is completely general. For example, a similar formula applies also to a unital resistance quantity that varies with temperature. And it should also be noted that in view of the underlying complete ordered semigroup framework it is possible to define the temporal (and spatial) derivatives $\frac{\mathrm{d} Q}{\mathrm{dt}}$ of unital quantities, needed in formulating differential equations. For the present, we defer consideration of derived unital quantities, even though these are by far the most important in applications ${ }^{16}$

\subsection{Semilinear spaces of unital base quantities}

The puzzling question of the continuum variety of unital quantities of a given kind, having the form of a complete ordered semigroup isomorphism $Q: \mathscr{Q} \longrightarrow \mathbb{R}_{+}$between the target system's quantity-constrained state space and the space of positive reals, may now be answered geometrically by representing quantities in terms of positive half-lines (rays), drawn from the Cartesian origin of the right quadrant, as displayed in Figure 1.

The Cartesian positive half-line bundle in Figure 1 illustrates all unital quantities relative to an arbitrarily chosen reference quantity $Q$ (represented by the positive half-diagonal) with its unit encoded by the unique state $q_{u}$ satisfying $Q\left(q_{u}\right)=$ 1. Similarly, there is the unitizing state $q^{\prime}$ for $Q^{\prime}$ obeying the unitizing condition $Q^{\prime}\left(q^{\prime}\right)=1$ and the unitizing state $q^{\prime \prime}$ for $Q^{\prime \prime}$ fulfilling the requirement $Q^{\prime \prime}\left(q^{\prime \prime}\right)=1$.

\footnotetext{
${ }^{16}$ The notion of time quantity requires more attention; it will be discussed in detail in the last part of this section.
}

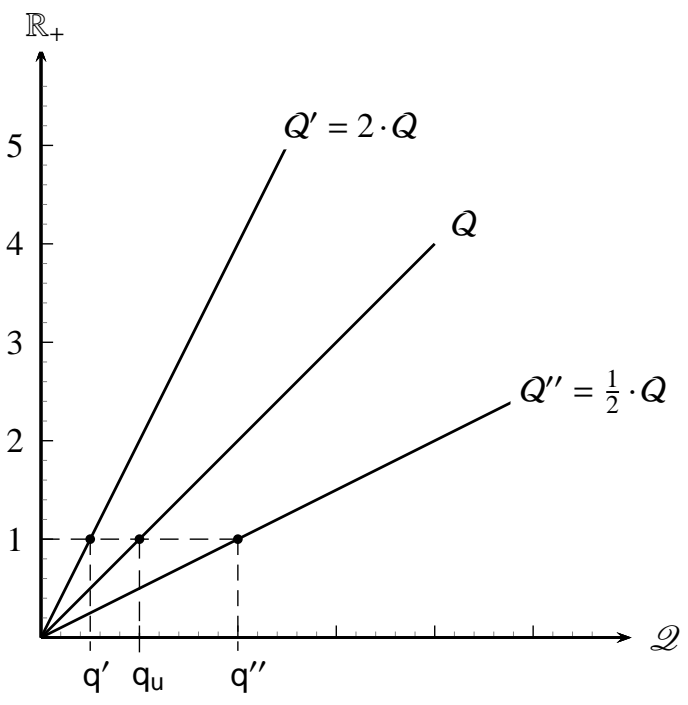

Fig. 1. Geometric depiction of the bundle of unital quantities of a given kind, relative to a reference quantity $Q$.

Now, because according to the unit-conversion formula $Q^{\prime}=\alpha \cdot Q$ any unital quantity $Q^{\prime}$ is uniquely determined by a designated or preferred base quantity $Q$, we can treat units in a dual manner by keeping the unitizing state implicit, fixed and secondary. For instance, in the case of length let the fixed state be $q_{m}$ for " 1 meter" (e.g., realized by the platinumiridium etalon). And instead of state-based reasoning, regard $Q$ as the base unital quantity that uniquely specifies all the other quantities of the same kind by the unit-change conversion

$$
Q^{\prime}=\frac{Q^{\prime}}{Q} \cdot Q
$$

Here we have chosen a special "torsor-theoretic" notation $\frac{Q^{\prime}}{Q}$ for the dimensionless conversion factor $\alpha$ (from $Q^{\prime}$ to $Q$ ) in $\mathbb{R}_{+}$that makes all base-change calculations remarkably efficient. $^{17}$

The following sequential base-change condition

$$
\left[Q^{\prime \prime}=\frac{Q^{\prime \prime}}{Q^{\prime}} \cdot Q^{\prime} \& Q^{\prime}=\frac{Q^{\prime}}{Q} \cdot Q\right] \Longrightarrow Q^{\prime \prime}=\frac{Q^{\prime \prime}}{Q} \cdot Q
$$

quantity $Q^{\prime}$ and $Q^{\prime}$ to $Q$, then we can certainly convert $Q^{\prime \prime}$ to $Q$ via the mediating quantity $Q^{\prime}$.

Obviously, we also have the equality

$$
\frac{Q_{1}}{Q_{2}}=\frac{\frac{Q_{1}}{Q}}{\frac{Q_{2}}{Q}}
$$

that allows to treat ratios of quantities without any reference to a chosen "unit" quantity.

\footnotetext{
${ }^{17}$ As we have seen, in Maxwell's framework the notion of unit is introduced in a dual (implicit) fashion in terms of the ratio $\frac{Q}{Q_{u}}$ of any quantity $Q$ of interest to a frozen reference quantity $Q_{\mathrm{u}}$ of the same kind, so that $Q=\frac{Q}{Q_{u}} \cdot Q_{u}$ holds. By way of concrete illustration, if $\mathcal{L}$ is a fixed unital length quantity, then we may think of the ratio $\frac{\mathcal{L}^{\prime}}{\mathcal{L}}$ as the "magnitude" in $\mathbb{R}_{+}$ of the length quantity $\mathcal{L}^{\prime}$ under consideration with respect to the "unit quantity" $\mathcal{L}$. In the Euclidean tradition of classical measurement, the empiricist ontology of real numbers is founded on the bedrock of ratios of quantities.
} 
Referring again to Figure 1, note that if we switch from unital quantity $Q$ to quantity $Q^{\prime}=2 \cdot Q$ that is twice as large, then the measurement results get divided by 2 , since $Q(q)=$ $\frac{1}{2} \cdot Q^{\prime}(q)$ for all states $q \in \mathscr{Q}$. So in this way the yard-unitized, inch-unitized and all the other length-unitized quantities can be uniquely converted into the primary meter-unitized quantity $\mathcal{L}$, and of course conversely, because theoretically any unital quantity can serve as a base.

Conversion factors give us an idea of how to proceed with setting up the algebraic structure of unital quantity calculus for quantities of a given kind. We already know that unital quantities possess a natural total order structure, defined by

$$
Q<Q^{\prime} \Longleftrightarrow 1<\frac{Q^{\prime}}{Q}
$$

satisfying the monotonicity condition $Q<Q^{\prime} \Longrightarrow Q+Q^{\prime \prime}<$ $Q^{\prime}+Q^{\prime \prime}$. Although it is simple to regard the set of all unital quantities of the same kind as an ordered semigroup with the customary addition operation $Q^{\prime}+Q^{\prime \prime}$ defined pointwise by

$$
\left[Q^{\prime}+Q^{\prime \prime}\right](\mathrm{q})=_{d f} Q^{\prime}(\mathrm{q})+Q^{\prime \prime}(\mathrm{q})
$$

for all states $q$ in $\mathscr{Q}$ or better yet, by recalling the definition from Section 1.1, from our point of view it is only natural to work in a significantly more powerful framework of strict (meaning without zero) one-dimensional semilinear spaces of unital quantities over the semifield $\left\langle\mathbb{R}_{+},+, \cdot, 1\right\rangle$ of strictly positive reals. Semilinear spaces are perfect analogs of vector spaces over the field of real numbers, except that the real scalar coefficients are restricted to positive real numbers. Therefore, from a formal standpoint, a semilinear space is just a variant of a positive cone in a vector space over reals.

We now turn our attention to the semilinear space (i.e., a strictly positive cone) of unital quantities of a given kind. Here the leading idea is that in order to study unital quantities of a certain kind, it is profitable to look at the space of all such quantities and tap into the wealthy technical resources of linear algebra - tensor product and quotient constructions in particular. Formally, we then have the following definition:

Definition 2 A semilinear space is a set $\mathbf{Q}$ (whose elements are called quantities) with two operations:

1. addition which assigns to each pair of quantities $Q, Q^{\prime}$ their $\operatorname{sum} Q+Q^{\prime}$, and

2. scalar multiplication which assigns to quantity $Q$ and each positive real number $\alpha$ the quantity $\alpha \cdot Q$.

These operations satisfy the following conditions:

(i) $Q+Q^{\prime}=Q^{\prime}+Q$.

(ii) $\left(Q+Q^{\prime}\right)+Q^{\prime \prime}=Q+\left(Q^{\prime}+Q^{\prime \prime}\right)$.

(iii) $\alpha \cdot\left(Q+Q^{\prime}\right)=\alpha \cdot Q+\alpha \cdot Q^{\prime}$.

(iv) $(\alpha+\beta) \cdot Q=\alpha \cdot Q+\beta \cdot Q$.

(v) $\alpha \cdot(\beta \cdot Q)=(\alpha \cdot \beta) \cdot Q)$.

(vi) $Q^{\prime}+Q=Q^{\prime \prime}+Q \Longrightarrow Q^{\prime}=Q^{\prime \prime}$.

(vii) $1 \cdot Q=Q$.
Because unital quantities of a given kind form a onedimensional semilinear space, they also satisfy the following transitivity property: For all $Q$ and $Q^{\prime}$ there exists an $\alpha>0$ such that

$$
Q^{\prime}=\alpha \cdot Q
$$

together with the freeness property

$$
\alpha \cdot Q=Q \Longrightarrow \alpha=1 \text {. }
$$

Since $\alpha$ in the transitivity condition is unique, it is convenient to symbolize it as a ratio (division) $\frac{Q^{\prime}}{Q}={ }_{d f} \alpha$.

In the case of length, the transitivity property says that the orbit of any unital length under the action of the semifield $\mathbb{R}_{+}$ of positive reals is the whole semilinear space $L$ and of course this one-dimensional space has no naturally distinguished elements. Any element $\mathcal{L}$ automatically generates the entire space L. In other words, the experimenter is free to move from any unital quantity whatsoever to any other unital quantity by simply considering the action of the semiring $\mathbb{R}_{+}$of positive reals on $\mathbf{L}$. Freeness means that precisely one action of $\mathbb{R}_{+}$will move a given unital length to another designated unital length. ${ }^{18}$

Remember that the space $\mathbf{Q}$ of unital quantities is linearly ordered. Some definitions of semilinear spaces include the default zero length quantity. Unfortunately, algebraic definitions often make too many concessions to the wellestablished linear algebra framework. In particular, it is possible to embed $\mathbf{Q}$ into a one-dimensional vector space over the real field $\mathbb{R}$. Here two things are worth noticing: (i) the classical vector space approach provides more structure than necessary in the sense that it includes too many empirically uninterpreted notions, such as negative length or negative mass, and (ii) any semilinear space $\mathbf{K}$ is naturally embeddable into its smallest $\mathbf{K}$-oriented linear space $\overline{\mathbf{K}}$ over the field of reals.

Recall again that unital quantities are complete ordered semigroup isomorphisms of the form $Q: \mathscr{Q} \longrightarrow \mathbb{R}_{+}$, illustrated by positive half-lines in Figure 1, and equipped with the earlier-defined addition and external scalar multiplication $\cdot: \mathbb{R}_{+} \times \mathbf{Q} \longrightarrow \mathbf{Q}$, specified pointwise by $[\alpha \cdot \mathbf{Q}](\mathbf{q})=\alpha \cdot \mathbf{Q}(\mathbf{q})$ for all states $q$ and positive reals $\alpha$ in $\mathbb{R}_{+} \cdot{ }^{19}$ The scalar action $\cdot: \mathbb{R}_{+} \times \mathbf{Q} \longrightarrow \mathbf{Q}$ of the semiring $\left\langle\mathbb{R}_{+},+, \cdot, 1\right\rangle$ of reals on $\mathbf{Q}$ represents all possible unit changes. To say that a unital quantity $Q$ of a given kind is equipped with a particular unit of measure simply means that it is a member of $\mathbf{Q}$. For this reason it is helpful to think of $\mathbf{Q}$ as a dimension-theoretic encoding of the kind or quantity type of $Q$. In dimensional algebra, onedimensional semilinear spaces $\mathbf{Q}$ of unital quantities (e.g., for

\footnotetext{
${ }^{18}$ An alternative formulation of the freeness property is $\alpha \cdot \mathcal{L}=\alpha^{\prime} \cdot \mathcal{L} \Longrightarrow$ $\alpha=\alpha^{\prime}$. A closely related treatment of the structure of $\mathbf{L}$ relies on the language of torsors or semiaffine spaces over the semiring $\mathbb{R}_{+}$of positive reals, but in this paper we do not need to consider these.

${ }^{19}$ The semilinear space $\mathbf{Q}$ is also a metric space under the distance function $\mathbf{d}\left(Q^{\prime}, Q^{\prime \prime}\right)=_{d f}\left|\frac{Q^{\prime}}{Q}-\frac{Q^{\prime \prime}}{Q}\right|$. It is trivial to check that the distance function does not depend on the choice of $Q$. Thus, if needed, we can also think of $\mathbf{Q}$ as a topological and hence a Borel measurable semilinear space. Alternatively, the order topology on $\mathbf{Q}$ is given by the subbasis family of subsets of the form $\left\{Q \mid Q<Q^{\prime}\right\}$ and $\left\{Q \mid Q^{\prime \prime}<Q\right\}$ for all $Q^{\prime}$ and $Q^{\prime \prime}$.
} 
length, time, mass and electric charge) are introduced from the start as basic higher-level (type-theoretic) undefined notions.

Physical theories are known to be based on a wide range of basic and derived quantity types, and operations thereon, even though they are usually formulated in measurement unitindependent ways. Keep in mind that intuitively the semilinear space $\mathbf{L}$ of unital length quantities can be viewed as the set of families of denominated (unitized) positive real numbers of various forms, including $\{\alpha$ meters $\mid \alpha>0\},\{\beta$ inches $\mid \beta>0\}$, $\{\gamma$ kilometers $\mid \gamma>0\}$, and so forth. Therefore, if the interest is in length values involving a specific measurement unit of length, e.g., given by the unital length quantity $\mathcal{L}$, then one can employ a less convenient alternative semilinear space notation $\mathbb{R}_{+} \mathcal{L}$ for $\mathbf{L}$ that explicitly displays the chosen unital quantity $\mathcal{L}$.

For example, the distance between any pair of points of a metric space $\mathscr{M}$ acquires a measurement-theoretic meaning only if it is encoded by a unital length-valued metric of the form $\mathbf{d}: \mathscr{M} \times \mathscr{M} \longrightarrow \mathbb{R}_{+} \mathcal{L}$. In this case the value $\mathbf{d}\left(m, m^{\prime}\right)$ of the unitized distance $\mathbf{d}$ between two distinct spatial points $m$ and $m^{\prime}$ is calculated directly in the measurement unit carried by $\mathcal{L}$, i.e., we have $\mathbf{d}\left(m, m^{\prime}\right)=\alpha \cdot \mathcal{L}$ for some $\alpha>0$, stating that the distance between the chosen points is given in measurement units specified by $\mathcal{L}$.

No conceptual problem exists here because choosing a unit for length is equivalent to choosing a unital length $\mathcal{L}$ in $\mathrm{L}$ with $\mathcal{L}^{\prime}=\frac{\mathcal{L}^{\prime}}{\mathcal{L}} \cdot \mathcal{L}$ for any other unital length $\mathcal{L}^{\prime}$. To avoid distracting measurement-theoretic details, mathematical physicists tend to cast their definitions of distance directly in terms of (unitless) real numbers rather than in terms of unitized lengths in L. More generally, the same custom applies to time, mass, electric current, and so forth. As we shall see, the semilinear space $\mathbf{T}$ of unital time quantities has far-reaching applications and so does the semilinear space $\mathbf{M}$ of unital mass quantities.

The following is a well-established strategy in mathematics: Any time a new object is defined, there should be a way of specifying how one of these objects transforms into another one in a structure-preserving manner. Accordingly, a map $\mathcal{H}: \mathbf{K} \longrightarrow \mathbf{K}^{\prime}$ from a semilinear space $\mathbf{K}$ to a semilinear space $\mathbf{K}^{\prime}$ is called a semilinear homomorphism provided that the equalities $\mathcal{H}\left(\boldsymbol{k}+\boldsymbol{k}^{\prime}\right)=\mathcal{H}(\boldsymbol{k})+\mathcal{H}\left(\boldsymbol{k}^{\prime}\right)$ and $\mathcal{H}(\alpha \cdot \boldsymbol{k})=\alpha \cdot \mathcal{H}(\boldsymbol{k})$ hold for all $\boldsymbol{k}, \boldsymbol{k}^{\prime} \in \mathbf{K}$ and $\alpha \in \mathbb{R}_{+}$. Cascade compositions of semilinear maps are semilinear.

It is well known and easy to show that the set of all semilinear homomorphisms from $\mathbf{K}$ to $\mathbf{K}^{\prime}$, denoted by $\operatorname{sLin}\left(\mathbf{K}, \mathbf{K}^{\prime}\right)$, is also a semilinear space. A one-to-one and onto semilinear homomorphism between two semilinear spaces is called a semilinear isomorphism. From now on we shall write $\mathbf{K} \cong \mathbf{K}^{\prime}$ if spaces $\mathbf{K}$ and $\mathbf{K}^{\prime}$ are semilinear-isomorphic.

We now turn to a list of useful elementary theorems characterizing the ratio $\frac{Q^{\prime}}{Q}$, defined by the unique positive real number $\alpha$ satisfying the equation $Q^{\prime}=\alpha \cdot Q$, i.e., we have $Q^{\prime}=\frac{Q^{\prime}}{Q} \cdot Q$.

Theorem 1 For all $Q, Q^{\prime}, Q^{\prime \prime}$ and $\alpha, \beta>0$ the following characterizations hold for all unital quantities: (i) $\frac{\alpha \cdot Q}{Q}=\alpha$.

(ii) $\frac{Q^{\prime}+Q^{\prime \prime}}{Q}=\frac{Q^{\prime}}{Q}+\frac{Q^{\prime \prime}}{Q}$.

(iii) $\frac{Q^{\prime}}{Q}=\left(\frac{Q}{Q^{\prime}}\right)^{-1}$.

(iv) $\frac{Q^{\prime}}{Q} \cdot Q=Q^{\prime}$.

(v) $\alpha \cdot \frac{Q^{\prime}}{Q}=\frac{\alpha \cdot Q^{\prime}}{Q}$.

(vi) $\frac{Q}{Q^{\prime}} \cdot \frac{Q^{\prime}}{Q^{\prime \prime}}=\frac{Q}{Q^{\prime \prime}}$.

(vii) $\frac{Q^{\prime \prime}}{Q^{\prime}} \cdot Q=\frac{Q}{Q^{\prime}} \cdot Q^{\prime \prime}$.

(viii) $\alpha \cdot Q=\alpha \cdot Q^{\prime} \Longrightarrow Q=Q^{\prime}$.

(ix) $\alpha \cdot Q=\beta \cdot Q \Longrightarrow \alpha=\beta$.

(x) $Q^{\prime}(\mathrm{q})=1 \Longleftrightarrow Q(\mathrm{q}) \cdot Q^{\prime}=Q$.

Since the proofs rest on completely elementary considerations, involving the definition of $\frac{Q^{\prime}}{Q}$ and appropriate substitutions, it seems safe to leave them as an exercise for the reader. However, to illustrate the proof format, we prove the last equivalence statement $(\mathrm{x})$ of the theorem.

Proof: From left to right, assume first that $Q^{\prime}(q)=1$ together with $Q^{\prime}=\alpha \cdot Q$ for some $\alpha>0$, so that $\alpha \cdot Q(q)=1$. Next, suppose by way of contradition that the conclusion of the statement is false, meaning $Q(q) \cdot Q^{\prime}\left(q^{\prime}\right) \neq Q\left(q^{\prime}\right)$ for some state $\mathrm{q}^{\prime} \neq \mathrm{q}$. Then we have at once the inequality $Q(\mathrm{q}) \cdot \alpha \cdot Q\left(\mathrm{q}^{\prime}\right) \neq$ $Q\left(q^{\prime}\right)$ and therefore the inequality $Q^{\prime}(q)=\alpha \cdot Q(q) \neq 1$, which is a contradiction.

For the converse, the evaluation of unital quantities in the assumed condition $Q(q) \cdot Q^{\prime}=Q$ at state $q$ immediately gives $Q^{\prime}(\mathbf{q})=1$.

Suppose that, for simplicity, we write $Q_{q}^{\prime}$ for quantity $Q^{\prime}$ unitized by state $q$, i.e., $Q_{q}^{\prime}(q)=Q^{\prime}(q)=1$ holds. Then the just proven result can be restated quite simply as the equality $Q_{\mathrm{q}}^{\prime}=\frac{1}{Q} \cdot Q$ and hence $\frac{1}{Q}=\frac{Q_{\mathrm{q}}^{\prime}}{Q}$.

What Theorem 1 shows is that we may treat the additive (minimal) part of quantity calculus by conceptualizing it in terms of ratios of quantities of the same kind. Below we will introduce a significantly more encompassing framework for derived unital quantities that incorporates the crucial product and quotient operations.

To formalize this much stronger framework in a mathematically robust and attractive manner, we will appeal to a simplified language of tensor products and quotients.

\subsection{Semilinear spaces of unital derived quantities}

The purpose of this subsection is to develop a rigorous theory of products and quotients of unital quantities, needed for the introduction of a great variety of derived quantities. It is a natural extension of quantity calculus discussed above. We will follow a path that will not lose the reader in the underbrush of complicated technicalities.

First we wish to draw a contrast between RTM's and our approach to derived quantities. With the exception of socalled conjoint measurement, in RTM there has not been a concerted and serious effort to develop a systematic qualitative structure-based setting for derived quantities - product 
and quotient quantities in particular. In RTM, this is not regarded as a serious problem, because once the representation theorems ensure the existence of certain homomorphisms encoding the needed base quantities, it is a straightforward matter to define all derived quantities in terms of suitable numerical functions of base quantities. RTM does not consider it necessary to analyze the structure of derived units and it does not find it important to ask when is a metrological statement true about a derived quantity's assumed value.

Our algebraic approach to derived unital quantities proceeds by defining the notion of their tensor product and that of a tensor quotient. These notions are then used to clarify and deepen the investigation of widely used notions of classical mechanics, such as mean velocity, unital density, and kinetic energy.

We now define the concept of tensor product of two semilinear spaces, which we have already hinted at above. It requires a bit of conceptual preparation that may be omitted without any significant loss of continuity.

Given any pair of unital quantities $Q: \mathscr{Q} \longrightarrow \mathbb{R}_{+}$and $Q^{\prime}$ : $\mathscr{Q}^{\prime} \longrightarrow \mathbb{R}_{+}$, not necesssarily of the same kind (e.g., one having the type of length and the other that of mass), we define their tensor product $Q \otimes Q^{\prime}$ by the composite of maps

$$
\mathscr{Q} \times \mathscr{Q}^{\prime} \stackrel{Q \times Q^{\prime}}{\longrightarrow} \mathbb{R}_{+} \times \mathbb{R}_{+} \stackrel{\cdot}{\longrightarrow} \mathbb{R}_{+}
$$

i.e., we set $\left[Q \otimes Q^{\prime}\right]\left(\mathrm{q}, \mathrm{q}^{\prime}\right)={ }_{d f} Q(\mathrm{q}) \cdot Q^{\prime}\left(\mathrm{q}^{\prime}\right)$ for all states $\mathrm{q}$ and $q^{\prime}$.

Let $\mathbf{Q} \otimes \mathbf{Q}^{\prime}$ denote the smallest semilinear space over positive reals, called a tensor product semilinear space, containing all of the above defined tensor product quantities $Q \otimes Q^{\prime}$ with $Q \in \mathbf{Q}$ and $Q^{\prime} \in \mathbf{Q}^{\prime}$. As the notion of a finitely generated semilinear space reveals, the associative addition operation in the tensor product space gives additively combined quantities of the form $Q_{1} \otimes Q_{1}^{\prime}+Q_{2} \otimes Q_{2}^{\prime}$ with arbitrary finite iterations and the scalar multiplication satisfies the following property: $\alpha \cdot\left[Q \otimes Q^{\prime}\right]=(\alpha \cdot Q) \otimes Q^{\prime}=Q \otimes\left(\alpha \cdot Q^{\prime}\right)$ for all $\alpha>0$.

By definition, the tensor product semilinear space of two semilinear spaces consists of finitary sums of elements of the form $\sum_{i} Q_{i} \otimes Q_{i}^{\prime}$ with $Q_{i} \in \mathbf{Q}$ and $Q_{i}^{\prime} \in \mathbf{Q}^{\prime}$.

However, because the tensor product of two onedimensional semilinear spaces is again a one-dimensional semilinear space, any of the elementary tensor quantities $Q \otimes Q^{\prime}$ generates the entire tensor product semilinear space. In more detail, as seen in the chain of equalities $Q_{1} \otimes Q_{1}^{\prime}+Q_{2} \otimes$ $Q_{2}^{\prime}=Q_{1} \otimes Q_{1}^{\prime}+\left(\alpha \cdot Q_{1}\right) \otimes\left(\beta \cdot Q_{1}^{\prime}\right)=(1+\alpha \cdot \beta) \cdot Q_{1} \otimes Q_{1}^{\prime}=Q \otimes Q_{1}^{\prime}$, addition of any pair of elementary tensor quantities automatically reduces to an elementary tensor quantity.

A common and useful way of obtaining more complicated tensor product quantities is via finitary iterations of binary products. However, this presents a slight technical problem in that instead of identities we only have the basic well-known and easy-to-check natural semilinear isomorphisms, generalizing the notions of commutativity and associativity:

\section{Theorem 2}

(i) $\mathbf{Q} \otimes \mathbf{Q}^{\prime} \cong \mathbf{Q}^{\prime} \otimes \mathbf{Q}$. (ii) $\left(\mathbf{Q} \otimes \mathbf{Q}^{\prime}\right) \otimes \mathbf{Q}^{\prime \prime} \cong \mathbf{Q} \otimes\left(\mathbf{Q}^{\prime} \otimes \mathbf{Q}^{\prime \prime}\right)$.

(iii) $\mathbf{Q} \otimes \mathbb{1} \cong \mathbf{Q} \cong \mathbb{1} \otimes \mathbf{Q}$.

Since the semilinear space $\mathbb{R}_{+}$of positive reals acts as a unit of the tensor product operation, accordingly the foregoing "unit" notation 1 for $\mathbb{R}_{+}$seems appropriate. Awkward as these tensor products are, they are all one-dimensional semilinear spaces, generated by a single elementary tensor quantity of the form $\left(Q \otimes Q^{\prime}\right) \otimes Q^{\prime \prime}$ or by its associative counterpart $Q \otimes\left(Q^{\prime} \otimes Q^{\prime \prime}\right)$.

Given a generating collection of one-dimensional semilinear spaces of unital quantities, viewed at a conceptually higher level as basic physical-geometric quantity types of lengh, time, mass, and so forth, we can construct from these semilinear spaces with the help of tensor products a large variety of semilinear spaces of derived unital quantities.

Now we will apply the preceding abstract tensor product construction to a single semilinear space of unital quantities $Q$. First, note that in this case the tensor product $Q \otimes Q^{\prime}$ with $Q$ and $Q^{\prime}$ in $\mathbf{Q}$ is commutative. That is to say, for some $\alpha$ we have $Q \otimes Q^{\prime}=Q \otimes(\alpha \cdot Q)=(\alpha \cdot Q) \otimes Q=Q^{\prime} \otimes Q$. By way of illustration, what this commutativity property says is that if the experimenter measures the base (width) of a rectangle in inches and its height in centimeters or the other way around, then he or she will get the same unitized area value. Of course, the area unit inch $\otimes$ centimeter is geometrically meaningful but admittedly not very common. The magnitude of the area remains the same independently of whether inches are converted into centimeters or conversely.

Because symmetric (commutative) tensor products are genuinely useful in their own right, in the commutative case we shall use the notations $Q \odot Q^{\prime}$ for $Q \otimes Q^{\prime}$ and $Q \odot Q$ instead of $\mathbf{Q} \otimes \mathbf{Q}$. Since in Section 1 we have already reserved the notation $Q \cdot Q^{\prime}$ for the commutative and associative product of quantities, we shall continue to use it also in place of the commutative tensor product $Q^{\prime} \odot Q$ of two quantities of the same kind. The only crucial difference between the classical Maxwellian approach and our approach to product quantities is that the classical approach aims at axiomatizing the product and we simply define it explictly, using the standard resources of multilinear algebra. As a result, products are not commutative in general, although they stand in an isomorphic relationship.

Next, note also that the point of the formal definition of the tensor product of the semilinear space of unital quantities with itself is to make the notions of such as area and volume quantities formally precise. In more detail, unital area quantities are elements of the symmetric product semilinear space $\mathbf{L} \odot \mathbf{L}$ and unital volume quantities belong to the space $\mathbf{L} \odot(\mathbf{L} \odot \mathbf{L})=(\mathbf{L} \odot \mathbf{L}) \odot \mathbf{L}$. It is common to define the tensorial power of a given semilinear space $\mathbf{Q}$ of quantities by iterating the tensor product of its copies: $\mathbf{Q}^{\odot n}={ }_{d f} \mathbf{Q} \odot \mathbf{Q} \odot \cdots \odot \mathbf{Q} n$ times. The direct sum of all of these tensorial powers defines the so-called tensor algebra $\mathbf{Q}^{\bullet}=\mathbb{1} \oplus \mathbf{Q} \oplus \mathbf{Q}^{\odot 2} \oplus \mathbf{Q}^{\odot 3} \oplus \cdots$ over $\mathbf{Q}$, in which the semilinear space $\mathbb{1}={ }_{d f} \mathbb{R}_{+}$acts as the unit of the tensor product. The conceptual role of a tensor algebra over the space of quantities of a given kind is to characterize all symmetric product quantities within a single algebraic 
framework. In the case of several spaces of base quantities, the generated tensor algebra takes care of the algebraic foundations for product-based quantity calculus.

At this point we have gone far enough to show how to construct a large variety of product unital quantities. We now come to one of the most important one-dimensional semilinear space constructions in the entire algebraic theory of unital quantities.

It is common knowledge that physicists use meter per second (in symbols $\mathrm{m} / \mathrm{s}$ ) as a typical derived measurement unit for the unitized mean velocity quantity. So now the question we have to ask is: Is there a well-defined semilinear space of unitized velocity quantities? The answer is in the affirmative, but first we have to make the idea concrete by introducing the fundamentally important notion of a quotient unital quantity, vital for the definition of a large variety of derived quantities.

A quotient unital quantity of quantity $Q$ of type (kind) $Q$, written $\frac{1}{Q}$, is a semilinear map of the form $\frac{1}{Q}: Q \longrightarrow \mathbb{R}_{+}$, defined by the ratio formula

$$
\left[\frac{1}{Q}\right]\left(Q^{\prime}\right)=\frac{Q^{\prime}}{Q}
$$

for all $Q^{\prime} \in \mathbf{Q}$. Since the addition and scalar operations on quotient quantities are defined pointwise, we may as well set $\mathbf{Q}^{-1}={ }_{d f}\left\{\frac{\mathbf{1}}{\mathbf{Q}} \mid \mathbf{Q} \in \mathbf{Q}\right\}$ for the semilinear space of all quotient unital quantities of type $\mathbf{Q}$.

There is an intimate connection between a semilinear space of quantities and its corresponding semilinear space of quotient quantities, given by the semilinear isomorphisms

$$
\mathbf{Q} \otimes \mathbf{Q}^{-1} \cong \mathbb{1} \cong \mathbf{Q}^{-1} \otimes \mathbf{Q},
$$

stating that quotient quantities are two-sided multiplicative inverses of quantities.

Based on the one-to-one correspondence between the set of conversion factors relative to quantity $Q$ and the space of unital quantities $\mathbf{Q}$, it should be clear that each quotient $\frac{1}{Q}$ establishes a semilinear isomorphism $\mathbf{Q} \cong \mathbb{R}_{+}$, where $\left\langle\mathbb{R}_{+},+, \cdot, 1\right\rangle$ is viewed as a semilinear space over itself. The functiontheoretic inverse of the quotient $\frac{\mathbf{1}}{Q}$ is the $\operatorname{map}\left(\frac{\mathbf{1}}{\mathbf{Q}}\right)^{-1}: \mathbb{R}_{+} \longrightarrow$ $\mathbf{Q}$ specified by $\left(\frac{\mathbf{1}}{\mathbf{Q}}\right)^{-1}(\alpha)=\alpha \cdot Q$.

Before we can discuss the subtleties of quotient quantities, we need to show the following simple characterization of quotients:

\section{Lemma 1}

$$
\frac{1}{\alpha \cdot Q}=\frac{1}{\alpha} \cdot \frac{1}{Q}
$$

Proof: Suppose the characterization is false.

Then $\frac{Q^{\prime}}{\alpha \cdot Q} \neq \frac{1}{\alpha} \cdot \frac{Q^{\prime}}{Q}$ for some $Q^{\prime}$ that has the form $Q^{\prime}=\beta \cdot Q$ for some $\beta$. Upon applying the clauses of Theorem 1 and a substitution we obtain the inequality $\frac{\beta \cdot Q}{\alpha \cdot Q} \neq \frac{\beta}{\alpha} \cdot \frac{Q}{Q}=\frac{\beta}{\alpha}$ and therefore the inequality $\beta \cdot Q \neq \frac{\beta}{\alpha} \cdot \alpha \cdot Q=\beta \cdot Q$, which is a contradiction and the proof is finished.

In anticipation of more general results, we note that the quotient construction can be combined with any finitedimensional real linear space $\mathbf{W}$ of vector quantities. For example, we have the real linear space $\mathbf{W} \otimes \mathbf{Q}^{-1}$ consisting of quotients of the form $\frac{\mathcal{W}}{Q}$, where $\left[\frac{\mathcal{W}}{Q}\right]\left(Q^{\prime}\right)={ }_{d f} \frac{Q^{\prime}}{Q} \cdot \mathcal{W}$ for all $Q^{\prime 20}$

The product construction also provides the onedimensional semilinear space $\mathbf{Q}^{\prime} \otimes \mathbf{Q}^{-1}$ defined by the tensor product of one-dimensional linear spaces $\mathbf{Q}^{\prime}$ and $\mathbf{Q}^{-1}$. In particular, the following important equalities hold in the tensor product space $\mathbf{Q}^{\prime} \otimes \mathbf{Q}^{-1}$ :

\section{Lemma 2}

(i) $\left(Q^{\prime}+Q^{\prime \prime}\right) \otimes \frac{1}{Q}=Q^{\prime} \otimes \frac{1}{Q}+Q^{\prime \prime} \otimes \frac{1}{Q}$.

(ii) $\alpha \cdot Q^{\prime} \otimes \frac{1}{\beta \cdot Q}=\frac{\alpha}{\beta} \cdot\left(Q^{\prime} \otimes \frac{1}{Q}\right)$.

Proof: These properties follow immediately from the definitions of product and quotient operations.

Inverse semilinear space constructions readily extend to symmetric tensor powers. For example, in the case of the length quantity type we have $\mathbf{L}^{-2}={ }_{d f} \mathbf{L}^{-1} \odot \mathbf{L}^{-1} \cong(\mathbf{L} \odot \mathbf{L})^{-1}$, and similarly for higher powers.

By now, the algebraic method of combining quantities using their products and quotients should be obvious.

As a rationality check, we now work through the details of the notion of unital mean velocity, defined earlier by the product $\mathcal{V}=_{d f} \mathcal{L} \otimes \frac{1}{\mathcal{T}}$, where $\mathcal{L}$ is a unital length quantity and $\frac{\mathbf{1}}{\mathcal{T}}$ refers to the quotient of the unital time quantity $\mathcal{T}{ }^{21}$

Before moving on to the investigation of unital velocities, we pause to include a bit of a detour into the structure of unital time quantities, underlying a broadly understood idea of temporal change in physical systems.

Conceptually diverging answers to questions such as "What is the time?" and "Will it take long?" immediately suggest that there are two basic approaches to the notion of physical time:

(i) The point-based or synchronic approach (we used earlier in discussing variable length) that focuses on the study of physical time based on the idea of temporal instances, intended to characterize the instantaneous occurrence of classical idealized point events in terms of temporal moments when they occur at any place in a threedimensional Euclidean simultaneity space. Typical examples of such events include flipping a light switch, collision of two particles, traffic light change, firing a gun, abutment of the respective leftmost (rightmost) tips of a target rod and a meter stick, and so forth.

\footnotetext{
${ }^{20}$ As may be expected by now, the inverse semilinear space construction $\mathbf{Q}^{-1}$ automatically extends to the inverse of the associated linear hull space $(\overline{\mathbf{Q}})^{-1}$

${ }^{21}$ It cannot be emphasized strongly enough that in order to be able to perform any kind of classical measurement of many derived physical quantities (e.g., velocity, acceleration and energy), first we must specify a designated Newtonian space-time coordinate system that fixes the simultaneous spatial location of the target system, measuring instrument, and experimenter, without significantly affecting the measurement operations. Of course, there is no privileged coordinate frame and the experimenter can select the one that best suits his or her measurement needs. Also, remember that experimenters situated in different coordinate frames will generally observe the target system in different shapes, sizes and states of motion. For example, since velocity of a moving particle has different values in different frames, so will its kinetic energy and all the other velocity-dependent quantities.
} 
(ii) The interval-based or diachronic approach that treats time in terms of durations (i.e., finitary non-zero lapses of time) of various interval events, happenings or spatial processes, confined to a finite bounded region of classical space. Often-discussed examples are earthquakes, solar or lunar eclipses, falling bodies, the cyclic behaviors of pendulums, quartz crystals, cesium atomic clocks and stopwatches.

These temporal ontologies are usually crafted in a mutually reductionist manner, so that durations can be viewed as finitary closed convex continuum-type subsets of the space of instants (with bona fide initial and terminal boundaries) and conversely, instants are thought of as constitutive elements of durations. Since clocks usually indicate time instants and measure temporal durations, we shall integrate these two approaches within a single classical one-dimensional oriented affine Euclidean framework.

Specifically, we will introduce a neo-Newtonian onedimensional oriented affine space $\boldsymbol{T}$ comprising time instants together with a structuring one-dimensional real linear space $\mathscr{T}$ of (closed) time intervals, future-oriented by its semilinear subspace (positive half-line) $\mathscr{T}_{+}$of positive (i.e., futureoriented) time intervals. ${ }^{22}$

Just as the classical length quantity is instantiated by rigid rods and other linear length-bearing physical bodies, the time quantity is realized by time-instantiating events. That is to say, by physical events that endure and thus are temporally localizable in terms of passage of elapsed time. Accordingly, events also fall into a basic twofold taxonomy:

(i) Instantaneous point events: These events are temporally localized at single time points. This is what we encounter when someone asks: exactly when did point event $\mathfrak{e}$ (e.g., an explosition) occur?

(ii) Interval events: These events are characterized by $d u$ rations, i.e., they are temporally localized by positive temporal intervals (time periods). In ordinary discourse, an interval event (e.g., a lunar eclipse or the cyclic behavior of a pendulum) is an objective physical happening in space-time (presumed to be independent of the experimenter's designated Galilean inertial coordinate frame), having a finite non-zero continuum duration, during which some participating physical objects or fields change their quantitative attributes.

By analogy between line segments and temporal intervals, two time intervals are temporally congruent when there is a

\footnotetext{
${ }^{22}$ It is well to emphasize that we are not identifying the selected mathematical structure of time with the objective physical time it purports to model and we are not assuming here that these two are "isomorphic" in any useful sense. To keep things simple, we regard physical space-time as something that exists in its own right and with its own manner, and is endowed with certain physical-geometric structures that classical neo-Newtonian models are able to capture to an acceptable degree of adequacy. We are ontologically committed only to space-time structures that are minimally required in characterizing quantities in the context of kinematics and dynanics of classical bodies, particles and fields.
}

temporal translation that uniquely translates the first interval into the second interval. Since temporal congruence is compatible with the ordinary composition of future-oriented temporal intervals (the addition of equivalence classes of two time intervals is given by the equivalence class of addition of their representatives), it is straightforward to justify that under the strict total shorter than temporal order relation < and the composition $*$ of two time intervals modulo temporal congruence, temporal intervals form a complete ordered semigroup.

So now a unital time quantity can be conceived as a complete ordered semigroup isomorphism of the form $\mathcal{T}: \mathscr{T}_{+} \longrightarrow$ $\mathbb{R}_{+}$, where $\mathscr{T}_{+}$serves as the space of temporal durations that physical events instantiate.

So as to suit the intended interpretation of unital time quantities, we now recall the basic measurement unit of time. As well known (see, e.g., page 19 in [10]), in SI the standard unit of time is 1 second and it is defined as follows:

The time quantity $\mathcal{T}$ is unitized by 1 second provided that the time interval $\tau_{\mathrm{sec}}={ }_{d f} \mathcal{T}^{-1}(1)$, modulo temporal congruence, is equal to the duration of a cesium (Cs 133) atom (at rest and at temperature 0 Kelvin in the ground state) to perform exactly 9,192,631,770 complete microwave oscillations.

Here as elsewhere, we use the upper case boldface letter $\mathbf{T}$ to denote the semilinear space of all unitized time quantities. Of course, this semilinear space is isomorphic to $\mathbf{L}$, but not naturally isomorphic, and therefore is without empirical significance.

We are now ready to say formally what is meant by the unitized time of a physical event. The lapsed time (duration) of the interval event e of interest, e.g., last year's lunar eclipse, measured in seconds, is given by the equation Time $(\mathrm{e})=\mathcal{T}(\tau)$, where $\mathcal{T}$ is the second-unitized time quantity and $\tau$ encodes the lapse of physical time during which the target event e actually endures.

With the definition of time quantity in hand, we now return to the notion of unital mean velocity. As has become clear, we need states to get numerical magnitudes. Armed with the notation for states, we can now write

$$
\mathcal{V}(\mathrm{s}, \tau)=\left[\mathcal{L} \otimes \frac{1}{\mathcal{T}}\right](\mathrm{s}, \tau)=\mathcal{L}(\mathrm{s}) \cdot \frac{1}{\mathcal{T}}\left(\mathcal{T}_{\tau}\right)=\mathcal{L}(\mathrm{s}) \cdot \frac{\mathcal{T}_{\tau}}{\mathcal{T}}=\frac{\mathcal{L}(\mathrm{s})}{\mathcal{T}(\tau)},
$$

where the equality $\frac{1}{\mathcal{T}(\tau)}=\frac{\mathcal{T}_{\tau}}{\mathcal{T}}$ is a substitutional instance of the last clause in Theorem 1 , in which $\mathcal{T}_{\tau}$ is assumed to be unitized by $\tau$, i.e., we have $\mathcal{T}_{\tau}(\tau)=1$.

We cannot conclude this subsection without mentioning the nature of truth makers of statements about velocity values. When we say that the straightline mean velocity of a projectile in a given Galilean coordinate frame is 15 meters in 3 seconds, what we mean is that (i) the projectile traverses a spatial interval of 15 meters, and (ii) the projectile's journey lasts for the time interval of 3 seconds. Thus, metrological propositions about the mean velocity of a moving object involve two kinds of truth-makers: spatial and temporal. The 
first kind instantiates the object's traversed spatial interval, and the second underwrites the object's temporal mode of existence during motion and thereby instantiates the time interval spent during its journey.

It is easy to see that reasoning similar to our analysis of velocity readily generalizes also to other derived quantities that rely on product and quotient operations.

Having established the basic properties of derived unital quantities, we now turn to a brief discussion of their measurement.

\section{Measurement from a QUantization Perspective}

The principal purpose of classical deterministic measurement operations is to determine the values of one or several quantities of interest, usually referred to as the target system's measurands.

In classical physics, a measurement process is commonly represented in terms of interaction between the measured system realizing the measurand's values and an analog measuring instrument (built for the measurand) in a controlled surrounding environment. During the interaction the states of these two systems evolve jointly, characterized by the deterministic dynamical laws of motion of the coupled system + instrument. At the end of the interaction the measuring apparatus is found in a final state in which the system's measurand and the instrument's so-called pointer or indicator quantity become strongly correlated. After the interaction, measurement of the quantity of interest is achieved only when the position of the coupled instrument's pointer or digital display has been observed and recorded, giving the experimenter a sufficiently accurate numerical information about the measured quantity's extant value. ${ }^{23}$

It is important to note in the present context that RTM makes no reference at all to the physical details of the measured and measuring system, their interaction, and associated measurement uncertainties. It models measurement in such a way that most of the detail of the measuring instrument is ignored and only the target system's to-be-measured quantifiable attribute is considered. And the same goes for derived measurement. If for some reason a measurand $Q$ is not measured directly but is known to be empirically related to other quantities, say, $Q_{1}, Q_{2}, \cdots, Q_{n}$, then its values may be handled with the help of a so-called model equation

$$
Q=\mathcal{M}\left(Q_{1}, Q_{2}, \cdots, Q_{n}\right),
$$

in which the model function $\boldsymbol{M}$ is a multinomial function on the real line, representing the presumed empirical relationship between the measurand and the listed input quantities.

In RTM, these quantities are not accompanied with any probability density functions, known to be crucial for encoding and evaluating statistical uncertainties of measure-

\footnotetext{
${ }^{23}$ In deterministic settings, classical physics assumes that all quantities have definite values at all times whether measured or not, and that ideally any value under consideration can be determined by measurement with arbitrarily high accuracy without significantly affecting the target system's extant state.
}

ment. From the standpoint of our algebraic framework, omission of discrete-valued pointer quantities, describing the measurement uncertainty-laden and the quantizing (discretizing) character of measuring systems, is the single greatest technical weakness in RTM's analysis of measurement operations.

The classical Maxwellian account of measurement of quantities also adopts the model equation approach. However, just like RTM, it too is not specifically concerned with measurement uncertainty and therefore statistical analyses of measurement play no role.

While it is true that a more encompassing description of measuring instruments is fundamental in explicit physical descriptions of the dynamics of measurement, in algebraic and statistical theories of measurement it is highly effective and perfectly sufficient to bring into play only the states and quantities of measured and measuring systems.

Although currently there is no single precise account of the structure and behavior of chains and networks of measuring systems, for a wide class of measurement situations the Guide to the Expression of Uncertainty in Measurement (acronymed GUM) in [11] recommends to analyze measurement processes and accompanying measurement uncertainties, realized by measuring systems, in terms of serially connected functional components, generally including sensors, transducers, amplifiers, modulators, transmission units and analog-to-digital interface display modules together with pertinent error sources.

For ease of presentation, we simplify the above-mentioned series of functional elements into a cascade pair of two major kinds of physical components together with their characterizing quantities: (i) a broadly understood physical instrument that may include a sensor, conditioner, amplifier, and possibly a longer list of other signal processing units described by appropriate continuous influence quantities, and (ii) an $A / D$ indicator, display device or other analong-to-digital (A/D) convertor unit. ${ }^{24}$ This kind of simplified bipartite conceptualization of measuring systems and their operation is illustrated by the block diagram in Figure 2 below.

As the block diagram above shows, measurand $Q$ together with a perturbing influence quantity $Q_{1}$ are the instrument's inputs, while $Q^{\prime}$ is the instrument's intermediary output quantity. Since instruments are physical systems and their quantities live in a continuous realm, traditional dynamical systems theory and signal theory are well-equipped to provide adequate characterizations of their structure and behavior.

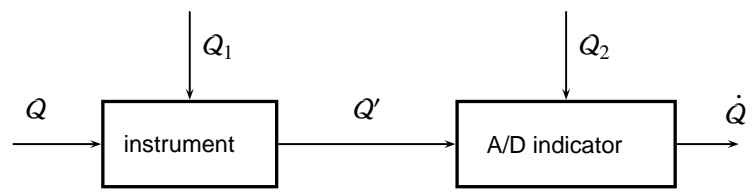

Fig. 2. A simplified schematization of measurement.

\footnotetext{
${ }^{24}$ Decomposition of a measuring system into its instrument and indicator components does not come out of thin air. It facilitates a crucial mathematical distinction between continuous and discrete-valued unital quantities.
} 
Some measurement theorists (e.g., [12]) seem to work under the standing assumption that dynamical models of instruments and statistics are fully sufficient for a complete account of measurement processes. Our approach to measurement operations deviates from the approach alluded to above in two ways: (i) we treat measurement operations from a strictly deterministic perspective, and (ii) in evaluating uncertainties (errors) we confine our attention to the A/D conversion and quantization aspects of measurement. In this setting, uncertainty is encoded by a round-off error, and this is how far deterministic quantity calculus can go without including probability density functions and defining quantities in terms of unitized random variables on measurable state spaces.

Recall that reading measurement outcomes relies on being able to sharply discriminate among the elements of a finite or countable collection of pointer values on the indicator's scale. In Figure 2, this idea is modeled by the $\mathrm{A} / \mathrm{D}$ convertor that receives the intermediary continuous unital quantity $Q^{\prime}$ jointly with a convertor error quantity $Q_{2}$ and outputs a quantized pointer quantity $\dot{Q}$. The measuring system's characterizing model equations are $Q^{\prime}=\mathcal{M}\left(Q, Q_{1}\right)$ and $\dot{Q}=\mathcal{M}^{\prime}\left(Q^{\prime}, Q_{2}\right)$.

These equations are extremely general and their measurement-theoretic content is not always clear. To cover a wide range of measurement applications, GUM recommends to view quantities as real-valued random variables accompanied with suitable probability density functions, and urges to use Bayesian inference methods in assessing measurement uncertainties. Because deterministic measurement operations occupy a place of special importance in classical physics and have an impressively wide scope, here we focus only on deterministic measurement operations, obtained by setting GUM's Bayesian probabilities to 0 or 1 .

Although many real-world measuring systems operate in complex ways, interestingly, often even very simple model equations, such as the linear equation $Q^{\prime}=Q+Q_{1}$, provide particularly good results. It is a remarkable fact that for a large collection of single-case static deterministic measurement procedures the conceptual issues can be overcome by adopting a surprisingly simple measurement model that directly links the system's states to the A/D indicator's pointer states.

This makes good sense when the effect of the instrument's influence quantity $Q_{1}$ is negligible relative the $\mathrm{A} / \mathrm{D}$ convertor's error quantity $Q_{2}$. In these measurement situations we may assume $Q^{\prime}=Q$ and analyze the measurement process entirely in the framework of analog-to-digital conversion. As we shall see, in this case the model function $\boldsymbol{M}^{\prime}$ is given by a so-called uniform mid-tread quantizer formula. Next, we move on to make these ideas concrete, using the simplest examples of measurement we can find. ${ }^{25}$

\subsection{Quantization models of measurement operations}

Quantization and direct deterministic measurement procedures are closely related. For some time it has been clear

\footnotetext{
${ }^{25}$ If the influence quantity $Q_{1}$ is not negligible, then the uniform mid-tread quantizer operator acts on the $\operatorname{sum} Q+Q_{1}$.
}

to some of the leading adherents of quantization theory (see, e.g., [19]) that quantization operations and (noiseless) singlecase deterministic measurement procedures performed by digital measuring instruments and by simple analog devices are structurally indistinguishable. Unfortunately, the implications of the engineers' quantization program for the foundations of measurement have not been properly explored. This subsection will be devoted to showing how quantization may be used to clarify and deepen our theoretical understanding of simple deterministic measurement procedures.

For purposes of illustration here and subsequently we shall consider two simple examples. Suppose we have a wellcalibrated digital ammeter that reads the measurement outcomes to two decimal places. It is intuitively plausible to assume that the ammeter comes with a deterministic uncertainty (quantization level) of $\varepsilon=0.01$ amps. Thus, if the ammeter shows a single digital reading of, say, 2.67 amps, then the proper way of reporting the electric current's actual value in the target circuit $c$ is to state that it is in the interval $[2.665,2.675)$. As hinted above, this kind of deterministic measurement uncertainty result fits perfectly well with the output of a so-called uniform mid-tred quantizer applied to a continuous input quantity. ${ }^{26}$

We hasten to add that in statistical approaches the readingscale error diagnosis is considerably more refined. Concretely, it is assumed that in the absence of any extra information the current's value is distributed in the interval $[2.665,2.675)$ with equal probability, encoded by a uniform (rectangular) probability density function (pdf). Therefore, under a Type B evaluation the so-called standard uncertainty, attributed to the noiseless quantized effect of measurement, is known to be given by the half of the width (i.e., 0.005 amps) of the interval divided by $\sqrt{3}$, so that the standard uncertainty is equal to 0.003 amps.

It is customary to express this result formally as $\dot{\mathcal{I}}_{\mathrm{c}}(\mathbf{i})=$ $2.67 \pm 0.003$, where $\dot{\mathcal{I}}_{\mathrm{c}}$ denotes the unitized electric current pointer quantity of measurand $\mathcal{I}_{\mathfrak{c}}$, measured in circuit $\mathfrak{c}$ and $\mathbf{i}$ refers to the pointer state instantiated by the ammeter.

Clearly, the statistical analysis of measurement uncertainty is significantly more reasonable than the deterministic method. However, uncertainty is also a matter of 'scientific rationality' that fits the intended application. In many measurement situations the issue is not using the "best available model" of unertainty but employing a simple model that is good enough for the practical case in question. ${ }^{27}$

\footnotetext{
${ }^{26}$ In general, deterministic uncertainty present in a single measurement performed by a single digital or analog instrument is given by the \pm -half of the least count of the measuring instrument. Naturally, measurement specialists are interested in uncertainties that are carefully tailored to the unital quantities they measure. For example, if unital distance were unitized and measured in light-years or in parsec units, then the assessment of associated measurement uncertainty in meters would be a poor choice. At the other extreme, if unital length were unitized and measured in the ångström unit, then the evaluation of measurement uncertainty in millimeters would be completely absurd.

${ }^{27}$ This kind of rationality issue becomes even more telling when one reflects on the ranges of application of Newtonian versus special and general relativistic mechanics.
} 
For the second example, suppose we measure the length of a straight rigid rod with a well-calibrated meter stick on which the smallest uniform reading scale divisions are given by thin uniform marks 1 millimeter apart. That is to say, the least count of the analog meter stick is 1 millimeter (i.e., using the earlier introduced notation we have $\varepsilon=0.001$ meters). Now, if the measurer reads the rod's length to be closest, say, to the $1,678^{\text {th }}$ millimeter mark in a single measurement under specified temperature and other relevant influence factors, then the actual value of the rod's length-in-millimeters must be somewhere in the numerical interval $[1,677.5,1,678.5)$. In the same manner as above, deterministic length measurement can be interpreted as a round-off type quantization operation (with quantization level 1 millimeter) that transforms a continuous unital length quantity into a discrete-valued unital length pointer quantity.

This approach works well in classical mechanics and related applications. The chief alternative to deterministic error evaluation is a statistical model that uses a triangular pdf over the numerical interval $[1,677.5,1,678.5)$. Here the idea is that the measurand's most probable value is given by the interval's midpoint and the values away from the midpoint in both directions are proportionately less likely.

So, concisely put, under a Type $B$ evaluation the so-called standard uncertainty, attributed to the quantized effect of measurement by a meter stick, is well known to be specified by the half of the width (i.e., 0.0005 meters) of the interval divided by $\sqrt{6}$, so that the standard uncertainty is equal to 0.0002 meters. It is customary to express this result as $\dot{\mathcal{L}}_{\mathrm{r}}(\mathbf{s})=1.678 \pm 0.0002$, where $\dot{\mathcal{L}}_{\mathrm{r}}$ denotes the length pointer quantity of rod $\mathrm{r}$ unitized by 1 meter, and $\mathbf{s}$ refers to the pointer state instatiated by the meter stick. Just as in the preceding example, in many practical applications a deterministic framework is fully sufficient for the assessment of uncertainty.

Now is a good time to return to the notion of state that we introduced earlier as a truth maker for metrological statements. To get clear on the truth or falsehood of statements about pointer quantity values, we need a legitimate notion of a pointer state, instantiated by measuring systems. We introduce this concept formally and examine its properties in some detail.

For purposes of direct deterministic measurement, we find it natural and adequate to define the measuring system's pointer states as suitable coarse-grained states of the measured system. We have seen in Section 2 that the measured system's state space for measurand $Q$ may be specified by a complete ordered semigroup $\langle\mathscr{Q}, \prec, \boldsymbol{\Psi}\rangle$.

To formalize the notion of state space of a measuring system designed to measure the unital quantity $Q: \mathscr{Q} \longrightarrow \mathbb{R}_{+}$of interest, we use an interval-based ordered uniform partition

$$
\left(0, q_{1}\right)<\left[q_{1}, q_{2}\right)<\left[q_{2}, q_{3}\right)<\cdots
$$

of the target system's state space $\langle\mathscr{Q}, \prec, \boldsymbol{\Psi}\rangle$ that characterizes the measuring system's indiscernibility of the measured system's topologically proximal states. ${ }^{28}$
Uniform partitions discussed above can easily be turned into a very familiar notion, namely a corresponding indiscernibility equivalence relation, defined by the biconditional

$\mathrm{q} \equiv \mathrm{q}^{\prime} \Longleftrightarrow \mathrm{q}, \mathrm{q}^{\prime} \in\left[\mathrm{q}_{n}, \mathrm{q}_{n+1}\right)$ for some natural number $n \geq 1$

or $\mathrm{q}, \mathrm{q}^{\prime}<\mathrm{q}_{1}$ for all states $\mathrm{q}$ and $\mathrm{q}^{\prime}$ in $\mathscr{Q}$. We are introducing indiscernibility relations in order to make precise the granularity of pointer states of measuring systems. Concretely, we define the measuring system's state space to be the quotient space $\mathscr{Q}_{/ \equiv}$ of $\mathscr{Q}$, modulo measurement indiscernibility, consisting of equivalence classes [q] of system states that the measuring system treats as indiscernible from $\mathrm{q}$. These instrument states encapsulate all information that is necessary and sufficient for determining the pointer quantity's values. Importantly, the induced projection map $\Pi: \mathscr{Q} \longrightarrow \mathscr{Q}_{/ \equiv}$, defined by $\Pi(\mathrm{q})=_{d f}[\mathrm{q}]$ for all $\mathrm{q}$, directly links the measured system's states to the measuring system's states and captures the crucial system-to-instrument causal dependence relationship.

Articulation of transition from a measuring system with finer-grained pointer states to that with coarser-grained pointer states is handled by the ordering of indiscernibility relations. Interestingly enough, the indiscernibility relation $\equiv$ is finer (i.e., encodes less or equal amount of uncertainty) than relation $\equiv^{\prime}$ just in case the condition

$$
q \equiv q^{\prime} \Longrightarrow q \equiv^{\prime} q^{\prime}
$$

holds for all system states $q$ and $q^{\prime}$.

For reasons of space, we cannot provide a detailed justification of why we use indiscernibility equivalence relations in modeling deterministic measurement operations. Very briefly, we use them with an eye on the fact that measuring systems come with a built-in deterministic uncertainty (specified in part by limited accuracy, resolution and other performance or design factors).

This means that measuring systems are not able to distinguish between two target system states that are topologically close to each other, and this is formally accommodated by the notion uniform equivalence relation on states. ${ }^{29}$ Keep in mind, however, that the qualitative notion of indiscernibility of states would not be very useful if it were posited without the existence of an empirically meaningful numerical representation. Fortunately, one of the hallmarks of any uniform indiscernibility relation (characterizing the approximate character of deterministic measurement) is its one-to-one correspondence with a numerical uncertainty $\varepsilon>0$, as shown in

\footnotetext{
${ }^{28}$ It should be noted that we use partitions of states made up of half-open intervals because without them we do not have a satisfactory conceptual tool for handling ordered partitions. The distinguishing feature of partitions we require is the uniformity of their cells. An interval-based partition is uniform provided that the partial subtractions $\mathrm{q}_{n+1} \dot{-} \mathrm{q}_{n}$, specified by the boundary states of all half-open intervals $\left[q_{n}, q_{n+1}\right)$, are the same and equal to $\frac{1}{2} \cdot q_{1}$. The sameness of subtraction states ensures the partition cell's uniformity.

${ }^{29}$ See [1] for a detailed discussion of discretization and additional references.
} 
the diagram of partitions below:

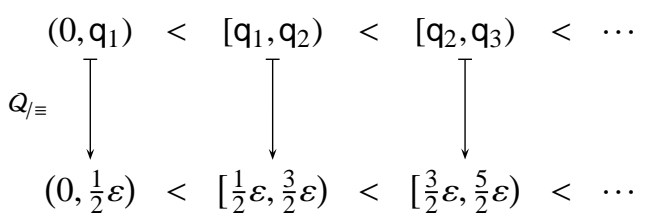

Suppose the measuring instrument's indiscernibility relation on the measured system's state space $\mathscr{Q}$ (in the context of measuring $Q$ ) is given by the ordered uniform partition, presented by the chain of half-open intervals, as depicted in the first row of the diagram above. We can represent this uniform indiscernibility relation numerically by setting $\varepsilon=2 Q\left(q_{1}\right)$ and $Q\left(\mathrm{q}_{n+1}\right)-Q\left(\mathrm{q}_{n}\right)=\varepsilon<1$ for all $n \geq 1$, where $\varepsilon$ is thought of as the measuring system's deterministic uncertainty (quantization level), inextricably tied to the measurement of $Q$. It is important to note that in the background of measuring quantity $Q$, the size of quantitative uncertainty $\varepsilon$ is determined by the granularity of the instrument's indiscernibility relation $\equiv .{ }^{30}$

By focusing on quantitative indiscernibility levels $\varepsilon$ we can accommodate any uniform partition of the measured system's state space that encodes the coarse-grained reading scale of the measuring system. Of course, partitions that fail to meet the uniformity requirement are not part of this model. In practice, it is natural and obvious to use measuring instruments with indiscernibility levels that are quite small relative to the measurand's unit.

So how does the quantitative indiscernibility level $\varepsilon$ account for the instrument's reading scale? It does so by specifying an equivalence relation $\equiv_{\varepsilon}$ on the set of positive reals, symbolized by the chain of half-open numerical intervals in the second row of the preceding diagram. The indiscernibility equivalence relation $\equiv_{\varepsilon}$ on the numerical ordered semigroup $\left\langle\mathbb{R}_{+},<,+\right\rangle$of quantity values can also be defined by the kernel biconditional as follows:

$$
\alpha \equiv_{\varepsilon} \beta \Longleftrightarrow \mathfrak{R}_{\varepsilon}(\alpha)=\mathfrak{R}_{\varepsilon}(\beta)
$$

for all $\alpha, \beta \in \mathbb{R}_{+}$. Here the so-called round-off function $\mathfrak{R}_{\varepsilon}$ : $\mathbb{R}_{+} \longrightarrow \varepsilon \mathbb{N}$ with $\varepsilon \mathbb{N}={ }_{d f}\{0, \varepsilon, 2 \varepsilon, 3 \varepsilon, \cdots\}$ is given by

$$
\mathfrak{R}_{\varepsilon}(\alpha)={ }_{d f} k \cdot \varepsilon \Longleftrightarrow \alpha \in\left[\left(k-\frac{1}{2}\right) \cdot \varepsilon,\left(k+\frac{1}{2}\right) \cdot \varepsilon\right)
$$

for all natural numbers $k \geq 1$ and $\mathfrak{R}_{\varepsilon}(\alpha)=0$ for all $\alpha<\frac{1}{2} \varepsilon$, i.e., $\mathfrak{R}_{\varepsilon}^{-1}(0)=\left[0, \frac{\varepsilon}{2}\right)$. It is easy to see that the round-off function always rounds up at the step edges, i.e., we have $\mathfrak{R}_{\varepsilon}\left(\left(k+\frac{1}{2}\right) \cdot \varepsilon\right)=(k+1) \cdot \varepsilon$.

As illustrated in the preceding diagram of aligned partitions, there is an obvious isomorphism $Q_{/ \equiv}: \mathscr{Q}_{/ \equiv} \longrightarrow \mathbb{R}_{+/ \equiv_{\varepsilon}}$ between the instrument's state space and the quotient space of reals, modulo equivalence relation $\equiv_{\varepsilon}$. Simply, we set $Q / \equiv([q])=_{d f}[Q(q)]_{\varepsilon}$ for all states $\mathbf{q}$, where $[\alpha]_{\varepsilon}$ denotes the $\varepsilon$-equivalence class of positive reals in $\mathbb{R}_{+/ \equiv_{\varepsilon}}$.

\footnotetext{
${ }^{30}$ Perhaps the most important relationship to notice here is the one-way implication $\mathrm{q} \equiv \mathrm{q}^{\prime} \Longrightarrow\left|Q(\mathrm{q})-Q\left(\mathrm{q}^{\prime}\right)\right|<\varepsilon$. We should keep in mind the transitivity property of $\equiv$ that the absolute value-based indistinguishability relation fails to possess.
}

Using the length measurement example again, recall that the states of length bearing objects are encoded by line segments modulo spatial congruence, and the pointer states of the meter stick are captured by the equivalence classes of lengthwise-proximal line segments that, relative to the characterizing least count (grain size) or quantization level $\varepsilon$, the meter stick instatiates or "reads" as if they were the same. In this way, if the least count of the meter stick were 1 millimeter and if two rigid rods were instatiating two line segments that differ lengthwise less than (say) 1 micrometer, then the measuring meter stick would not be able to tell them apart.

Now the crucial step for our purposes is the introduction of the pointer quantity

$$
\mathscr{Q}_{/ \equiv} \stackrel{\dot{Q}_{\varepsilon}}{\longrightarrow} \varepsilon \mathbb{N}
$$

associated with measurand $Q$. This notion is firmly entrenched in the everyday practice of measurement and we define it as follows:

$$
\dot{Q}_{\varepsilon}([\mathrm{q}])= \begin{cases}k \varepsilon, & \text { if }\left(k-\frac{1}{2}\right) \varepsilon \leq Q(\mathrm{q})<\left(k+\frac{1}{2}\right) \varepsilon \text { with } k \geq 1 \\ 0, & \text { if } Q(\mathrm{q})<\frac{1}{2} \varepsilon\end{cases}
$$

Recall that the pointer quantity's value space $\varepsilon \mathbb{N}=d f$ $\{0, \varepsilon, 2 \varepsilon, 3 \varepsilon, \cdots\}$ consists of natural number multiples of the quantization level (least significant bit) $0<\varepsilon<1$, interpreted as the instrument's deterministic uncertainty. We know that when a skillful measurer wishes to measure a rod's length with a meter stick to the nearest millimeter, he or she typically rounds off the displayed value on the meter's scale to the closest millimeter mark. Thus, the rod's actual length will be off by a small amount, not exceeding $\frac{1}{2} \varepsilon$ millimeters.

It is useful to bear in mind that the numerical counterpart of pointer quantity $\dot{Q}_{\varepsilon}$ is the earlier introduced round-off function $\mathfrak{R}_{\varepsilon}: \mathbb{R}_{+} \longrightarrow \varepsilon \mathbb{N}$. Now that we have a good understanding of the notion of a measurand's pointer quantity, we can illustrate its relationship to the measurand geometrically by the deterministic error diagram shown in Figure 3 below.

In Figure 3, measurand $Q$ is represented by the diagonal in the positive quadrant of the Cartesian plane and the discretevalued pointer quantity $\dot{Q}_{\varepsilon}$ is its nonlinear measurement approximation, having the geometric form of a staircase. The shaded area around $Q$ represents the $\pm \frac{1}{2} \cdot \varepsilon$ deterministic quantization error range of measurement.

Here we have run quickly past a number of uncertainties that would have to be addressed in a longer account. For example, we have not considered (i) the offset error which is constant accross the input range of $Q$, (ii) the measuring system's noise $Q_{1}$ generated within the instrument, and (iii) the nonlinearity error caused by the imperfections of the A/D convertor.

All of these and other uncertainties affect the total measurement uncertainty in the form of percentages and make the relationship between the measurand's values and that of its pointer quantity considerably more involved. 


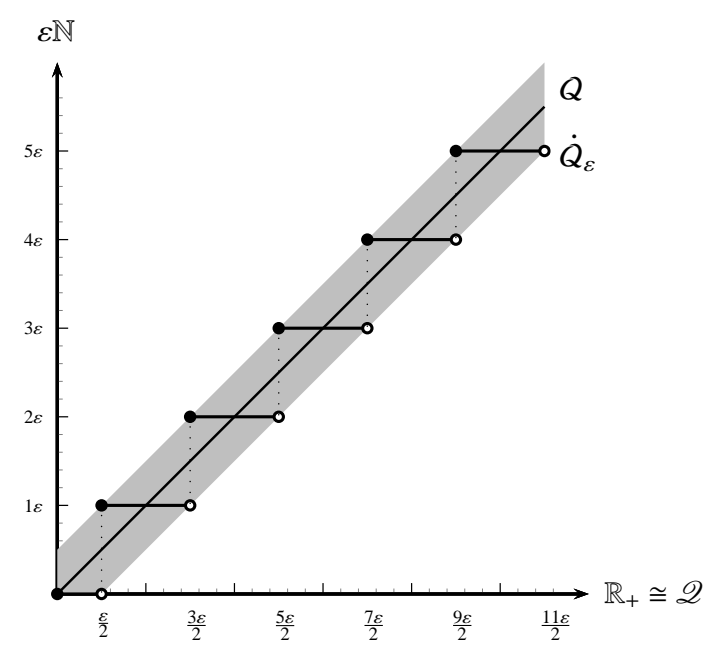

Fig. 3. The shaded uncertainty (error) zone of measurand $Q$, based on reading the values of pointer quantity $\dot{Q}_{\mathcal{E}}$.

The following commutative diagram highlights the basic relationships between quantities and round-off maps we have discussed so far.

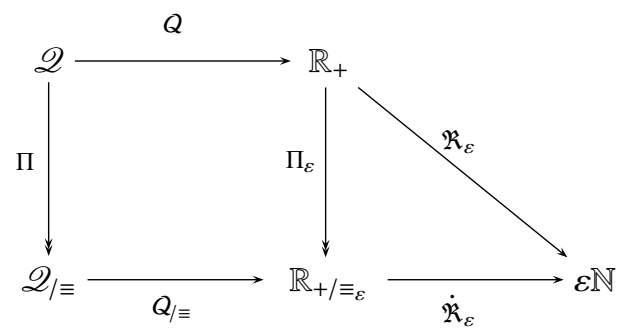

Note that in the diagram the pointer quantity is given in terms of composition of two mappings: $\dot{Q}_{\varepsilon}=\dot{\mathfrak{R}}_{\varepsilon} \circ Q_{/ \equiv}$, where $\dot{\mathfrak{R}}_{\varepsilon}\left([\alpha]_{\varepsilon}\right)=\mathfrak{R}_{\varepsilon}(\alpha)$ for all positive reals $\alpha$. The square in the diagram encodes the equality $Q_{/ \equiv}([\mathrm{q}])=\Pi_{\varepsilon}(Q(\mathrm{q}))$ for all $\mathrm{q}$. And the triangle captures the equality $\mathfrak{R}_{\varepsilon}(\alpha)=\dot{\mathfrak{R}}_{\varepsilon}\left([\alpha]_{\varepsilon}\right)$ for all $\alpha$.

The third commutative condition

$$
\dot{Q}_{\mathfrak{f}}([\mathrm{q}])=\mathfrak{R}_{\varepsilon}\left(Q_{\mathfrak{f}}(\mathrm{q})\right)
$$

in the diagram plays a crucial semantic role in specifying the truth conditions of measurement statements about the pointer quantity's value, obtained by measuring the value of measurand $Q$, instatiated by the target object $\mathfrak{f}$. The idea is to transfer the measurand's truth conditions to that of its pointer quantity with the help of the instrument's roundoff function.

In light of the above discussion of ordered partitions and indiscernibility relations, it is important to stress that in view of the uniformity of interval-based partitions, each half-open interval $\left[q_{n}, q_{n+1}\right)$ uniquely determines and is uniquely determined by the interval's midpoint state defined by $\mathrm{q}_{n} \mid \mathrm{q}_{n+1}=d f$ $\frac{1}{2}\left(q_{n}+q_{n+1}\right)$, commonly representing the "expected" state. In particular, it is easy to see that the midpoint of the corresponding numerical interval $\left[\left(n-\frac{1}{2}\right) \varepsilon,\left(n+\frac{1}{2}\right) \varepsilon\right)$ is $\left(n-\frac{1}{2}\right) \varepsilon \mid(n+$ $\left.\frac{1}{2}\right) \varepsilon=n \varepsilon$.
We can shed more light on what is going on here by noting that the pointer quantity value space $\langle\varepsilon \mathbb{N},<,+\rangle$ and the space $\left\langle\mathbb{R}_{+/ \equiv_{\varepsilon}},<+\right\rangle$ of equivalence classes induced by $\equiv_{\varepsilon}$ are both countable ordered semigroups. Moreover, the measuring instrument's state space $\left\langle\mathscr{Q}_{\mid \equiv},<,+\right\rangle$ is also a countable ordered semigroup. Regarding its structure, the total order $<$ is obvious and the addition operation is defined in $\mathscr{Q}_{/ \equiv}$ with the help of the midpoint operation. Specifically, the addition of two intervals is given by the interval determined by the sum of their midpoints. For example, the left endpoint of the sum $\left[q_{n}, q_{n+1}\right)+\left[q_{m}, q_{m+1}\right)$ is given by the state $Q^{-1}\left[Q\left(q_{n}\left|q_{n+1}+q_{m}\right| q_{m+1}\right)-\frac{1}{2} \varepsilon\right]$. The right endpopint is specified in a similar way. Because all three ordered semigroups are isomorphic to the ordered semigroup $\langle\mathbb{N},<,+\rangle$ of natural numbers, they are also pairwise isomophic. As a result, we obtain a simple discrete algebraic framework in which the $\mathrm{A} / \mathrm{D}$ convertor measurement error-laden outputs can be effectively studied. ${ }^{31}$

The question now arises whether the system-to-instrument projection map $\Pi: \mathscr{Q} \longrightarrow \mathscr{Q}_{/ \equiv}$, modeling a deterministic measurement of $Q$, is a homomorphism in any useful sense. Here our aim is to characterize a passage from the universe of continuous states and quantities to that of discrete pointer states and quantities. In giving a rigorous account of this conceptual shift from more structure to less structure we can properly understand the loss of information about quantity values due to measurement.

Clearly, upon passing to pointer states, we have to surrender our earlier commitment to strict ordering relations and switch to weak orderings. As a tradeoff, under this somewhat impoverished order structure the projection map remains monotone. That is to say, the monotonicity condition

$$
q \leq q^{\prime} \Longrightarrow[q] \leq\left[q^{\prime}\right]
$$

holds for all states $q$ and $\mathrm{q}^{\prime}$ in $\mathscr{Q}$, where, as before, we set $\Pi(q)=[q]$.

In like manner, one might wonder about the projection map's conservation of addition. Length measurement in carpentry provides a useful illustration. We are well aware that when carpenters build wooden structures from various pieces of boards by adding their measured widths, they tend to treat width-measurement errors as negligible. But of course we also know that carpenters typically measure the width of a board with some nonzero uncertainty. Apparently, the carpenters' error-laden measurement practices in adding boards do not significantly affect the quality of their work so long as there are only few boards to add accompanied with small errors, so that the total sum of uncertainties does not exceed the limit allowed by the construction. So the problem posed by the conservation of addition in measurement can be resolved by considering a partial semigroup addition of instantiated states that is defined only on the subset of the state space in which addition does not exceed the allowed threshold of uncertainty.

\footnotetext{
${ }^{31}$ See [3] and references therein for more detail on uncertainty in deterministic systems.
} 
In formal terms, the additivity law $\left[q+q^{\prime}\right]=[q]+\left[q^{\prime}\right]$ governing the instrument states generally fails and therefore so does the additive property of the composite map $\dot{Q}_{\varepsilon} \circ \Pi$ : $\mathscr{Q} \longrightarrow \varepsilon \mathbb{N}$. To appreciate the problem, note that in the case of adding $\mathfrak{b}+\mathfrak{b}^{\prime}$ two boards $\mathfrak{b}$ and $\mathfrak{b}^{\prime}$, the width discrepancy between them is relatively small, given by the error bound

$$
\left|\dot{Q}_{\mathrm{b}+\mathrm{b}^{\prime}}\left(\left[\mathrm{q}+\mathrm{q}^{\prime}\right]\right)-\dot{Q}_{\mathrm{b}}([\mathrm{q}])-\dot{Q}_{\mathrm{b}^{\prime}}\left(\left[\mathrm{q}^{\prime}\right]\right)\right| \leq \varepsilon
$$

Unfortunately, the effect of adding more boards leads to potentially larger errors. Concretely, if we consider three summands as in the pointer state $\left[q+q^{\prime}+q^{\prime \prime}\right]$, then the error bound jumps to $2 \varepsilon$, and so forth. To simplify our notation, we write $\dot{Q}$ for the pointer quantity of $Q$, in which the subscript $\varepsilon$ is temporarily dropped.

But why should we care about the conservation of addition by the composition $\dot{Q} \circ \Pi$ of the pointer quantity followed by the projection map? Because we know from Section 1 that the crucial prerequisite for handling the values of unital quantities instantiated by composite systems is the sum of the respective states of their constituents. So from the standpoint of modeling measurement operations, we are better off in a framework that includes addition.

Despite the foregoing lack of semigroup addition, we can significantly increase the flexibility of our approach to deterministic measurement by considering a partial semigroup addition operation of the form $\dot{+}: \mathscr{Q} \times \mathscr{Q} \longrightarrow \mathscr{Q}$ that is defined only on a suitable subset of so-called summable pairs of states - proximal to the partition intervals' midpoint states. This is the operation carpenters and engineers rely on when they combine their measurement data additively.

Another good reason for employing a partial semigroup addition in our measurement models is that with respect to it the indiscernibility relation $\equiv$ is a partial congruence relation.

It turns out that the problem of summability of states can be handled by a stipulative definition of a commutative summability relation $q \rrbracket q^{\prime}$ on $\mathscr{Q}$, stating that the states $q$ and $q^{\prime}$ are summable. To save space, we introduce the auxiliary notion of summability by the biconditional

$$
\mathrm{q}\left[\mathrm{q}^{\prime} \Longleftrightarrow\left[\mathrm{q}+\mathrm{q}^{\prime}\right]=[\mathrm{q}]+\left[\mathrm{q}^{\prime}\right]\right.
$$

for all states $q$ and $q^{\prime}$. As we see, the scope of summability of the measured system's states is determined by the measuring system's quantization level. We can now define the concept of partial addition of states quite simply as follows: $\mathbf{q}+\mathbf{q}^{\prime}=d f$ $\mathrm{q}+\mathrm{q}^{\prime}$, if $\mathrm{q} \rrbracket \mathrm{q}^{\prime}$, and undefined otherwise.

Upon weakening the usual notion of addition + on $\mathscr{Q}$ to a partial addition $\dot{+}$, we can accommodate in a unified way the incompleteness or partialness of information provided by measurement about measurands. In more detail, given a partial addition on the states of the measured system, the composite $\dot{Q} \circ \Pi:\langle\mathscr{Q}, \leq, \dot{+}\rangle \longrightarrow\langle\varepsilon \mathbb{N}, \leq,+\rangle$ of the measurand's pointer quantity and projection, mapping the measured system's states directly to the instrument's quantized numerical outputs, can now be viewed as a weak partial semigroup homomorphism satisfying the following conditions: (a) Weak monotonicity:

$$
\mathrm{q} \leq \mathrm{q}^{\prime} \Longrightarrow \dot{Q}([\mathrm{q}]) \leq \dot{Q}\left(\left[\mathrm{q}^{\prime}\right]\right)
$$

(b) Partial additivity:

$$
\dot{Q}\left(\left[q+\mathrm{q}^{\prime}\right]\right)=\dot{Q}([\mathrm{q}])+\dot{Q}\left(\left[\mathrm{q}^{\prime}\right]\right)
$$

In the light of these properties we see that the relationships between the world of continuous states and quantities and that of the associated discrete pointer states and quantities can be described by mappings that preserve the pertinent structures only weakly and partially. As we saw above, based on indiscernibility and uncertainties added by measurement, partial structures supply the correct formal tools for investigating the ever-present loss or incompleteness of information about the measurand's extant values.

With these comments, we bring our present investigation of quantities and their measurement to a close.

\section{Concluding Remarks}

In this paper we developed a simple and effective algebraic framework for quantity calculus and supervening deterministic measurement operations. The calculus is based on unitized quantities and accompanying state spaces that underly the truth conditions for metrological statements. In our investigation of the structure of quantities we used length, time, electric current, and mean velocity as primordial examples. For simplicity's sake and for reasons of space, we restricted our analysis to the case of deterministic measurement processes.

We have also addressed the algebraic nature of pointer states and pointer quantities characterizing measuring instruments, together with tightly connected measurement uncertainties. In bridging the gap between what experimenters regard as theoretical and what they take to be as measurementbased, we discussed the formal relationships between measured unital quantities and their associated pointer quantities.

There are vast areas of the subject of quantity calculus and measurement uncertainty which remain unexplored, including probabilistic and stochastic extensions, built over measurable state spaces, random quantities and their probability density functions. We intend to take up these topics in the near future.

\section{REFERENCES}

[1] Batitsky, V., Domotor, Z. (2007). When good theories make bad predictions. Synthese, 157, 79-103.

[2] de Boer, J. (1994/95). On the history of quantity calculus and the international system. Metrologia 31 (6), 405429.

[3] Domotor, Z. (2008). Structure and indeterminacy in dynamical systems. In Indeterminacy: The Mapped, the Navigable, and the Uncharted. MIT Press, 171-193. 
[4] Domotor, Z., Batitsky, V. (2010). An algebraic-analytic framework for measurement theory. Measurement, 43 (9), 1142-1164.

[5] Domotor, Z. (2012). Algebraic frameworks for measurement in the natural sciences. Measurement Science Review, 12, 213-233.

[6] Finkelstein, L. (2003). Widely, strongly, and weakly defined measurement. Measurement, 34, 39-48.

[7] Griesel, H. (1969). Algebra und Analysis der Größensysteme. Mathematisch-Physikalische Semesterberichte, 16 (1), 56-93 + 189-224.

[8] Hölder, O. (1901). Die Axiome der Quantität und die Lehre vom Mass. Berichte über die Verhandlungen der Königlichen Sächsischen Gesellschaft der Wissenschaften zu Leipzig, Mathematische-Physische Klasse, 53, 1-64.

[9] International Standards Organization. (1992). Quantities and Units - Part 0: General Principles. ISO 310:1992.

[10] BIPM. (2006; updated in 2014). SI Brochure: The International System of Units (SI), 8th edition. http://www.bipm.org/en/si/si-brochure/.

[11] International Organization for Standardization. (2011). Guide to the Expression of Uncertainty in Measurement (GUM). JCGM 102:2011.

[12] Ishikawa, S. (2006). Mathematical Foundations of Mea- surement Theory. Tokyo, Japan: Keio University Press.

[13] Krantz, D.H., Luce, R.D., Suppes, P., Tverskym, A. (1971). Foundations of Measurement, Vol. 1. Academic Press.

[14] Luce, R.D., Suppes, P. (2002). Representational measurement theory. In Handbook of Experimental Psychology, 3rd ed. Wiley, Vol. 4, 1-41.

[15] Suppes, P., Krantz, D.H., Luce, R.D., Tversky, A. (1989). Foundations of Measurement, Vol. 2. Academic Press.

[16] Suppes, P., Zinnes, J.L. (1963). Basic measurement theory. In Handbook of Mathematical Psychology. Wiley, Vol. 1, 1-76.

[17] Truesdell, C. (1977). A First Course in Rational Continuum Mechanics. Vol. 1 : General Concepts. Academic Press.

[18] BIPM. (2012). International Vocabulary of Metrology - Basic and General Concepts and Associated Terms (VIM 3rd edition). JCGM 200:2012.

[19] Widrow, B., Kollár, I. (2008). Quantization Noise: Roundoff Error in Digital Computation, Signal Processing, Control, and Communications. Cambridge University Press.

Received March 6, 2016. Accepted May 26, 2016. 